\title{
LncFZD6 initiates Wnt/ $\beta$-catenin and liver TIC self-renewal through BRG1-mediated FZD6 transcriptional activation
}

\author{
Zhenzhen Chen ${ }^{1} \cdot$ Yanfeng Gao ${ }^{1,2} \cdot$ Lintong Yao ${ }^{1} \cdot$ Yating Liu $^{1} \cdot$ Lan Huang $^{3} \cdot$ Zhongyi Yan $^{1} \cdot$ Wenshan Zhao ${ }^{1} \cdot$ \\ Pingping $\mathrm{Zhu}^{1} \cdot$ Haibo Weng ${ }^{1}$
}

Received: 1 September 2017 / Revised: 27 November 2017 / Accepted: 2 February 2018 / Published online: 14 March 2018

(c) The Author(s) 2018. This article is published with open access

\begin{abstract}
Liver tumor-initiating cells (TICs), the drivers for liver tumorigenesis, accounts for liver tumor initiation, metastasis, drug resistance and relapse. Wnt/ $\beta$-catenin signaling pathway emerges as a critical modulator in liver TIC self-renewal. However, the molecular mechanism of $\mathrm{Wnt} / \beta$-catenin initiation in liver tumorigenesis and liver TICs is still elusive. Here, we examined the expression pattern of 10 Wnt receptors (FZD1-FZD10), and found only FZD6 is overexpressed along with liver tumorigenesis. What's more, a divergent lncRNA of FZD6, termed lncFZD6, is also highly expressed in liver cancer and liver TICs. LncFZD6 drives liver TIC self-renewal and tumor initiation capacity through FZD6-dependent manner. LncFZD6 interacts with BRG1-embedded SWI/SNF complex and recruits it to FZD6 promoter, and thus drives the transcriptional initiation of FZD6 by chromatin remodeling. WNT5A, a ligand of FZD6, is highly expressed in liver nonTICs and drives the self-renewal of liver TICs through IncFZD6-BRG1-FZD6-dependent manner. Through FZD6

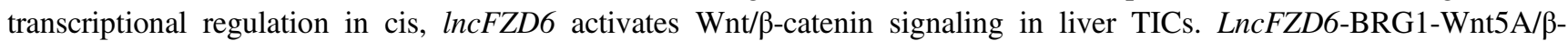
catenin pathway can serve as a target for liver TIC elimination. Altogether, lncFZD6 promotes Wnt/ $\beta$-catenin activation and liver TIC self-renewal through BRG1-dependent FZD6 expression.
\end{abstract}

\section{Introduction}

Liver cancer is one of the most serious cancers in the world. Liver cancer contains two common tumor types,

These authors contributed equally: Zhenzhen Chen, Yanfeng Gao.

Electronic supplementary material The online version of this article (https://doi.org/10.1038/s41388-018-0203-6) contains supplementary material, which is available to authorized users.

Zhenzhen Chen

chenzz2015@zzu.edu.cn

$\bowtie$ Pingping Zhu

pingpingchustc@hotmail.com

$\triangle$ Haibo Weng

whb@zzu.edu.cn

1 School of Life Sciences, Zhengzhou University, Zhengzhou 450001, China

2 Collaborative Innovation Center of New Drug Research and Safety Evaluation, Zhengzhou 450001 Henan Province, China

3 The First Affiliated Hospital, Zhengzhou University, Zhengzhou 450052, China hepatocellular carcinoma (HCC) and cholangiocarcinoma (CC). Heterogeneity is a major characteristic of liver cancer and largely increases the difficulty for clinical elimination [1]. Actually, there are several kinds of cells in liver samples. Liver tumor-initiating cells (TICs), a small subset cells within liver tumor bulk, account for liver tumor initiation, metastasis and relapse [2,3]. Liver TICs have the capacities of self-renewal and differentiation [4]. They generate various cell types and can propagate to refuel their population during tumorigenesis. According to these capacities, some functional assays were developed: sphere formation for self-renewal, side population for drug resistance, xenograft for propagation, gradient xenograft for tumor initiation and so on [5-7]. Recently, more and more surface markers of liver TICs have been discovered, including CD133, CD13, CD24, EPCAM and so on [8-10]. Other than surface marker, several transcription factors are also involved in liver TIC self-renewal, including c-Myc, Oct4, Zic2, Sox4 and so on [11, 12]. However, the biological characteristics of liver TICs remain elusive.

Several signaling pathways participate in liver TIC selfrenewal, including Wnt/ $\beta$-catenin signaling, Notch 

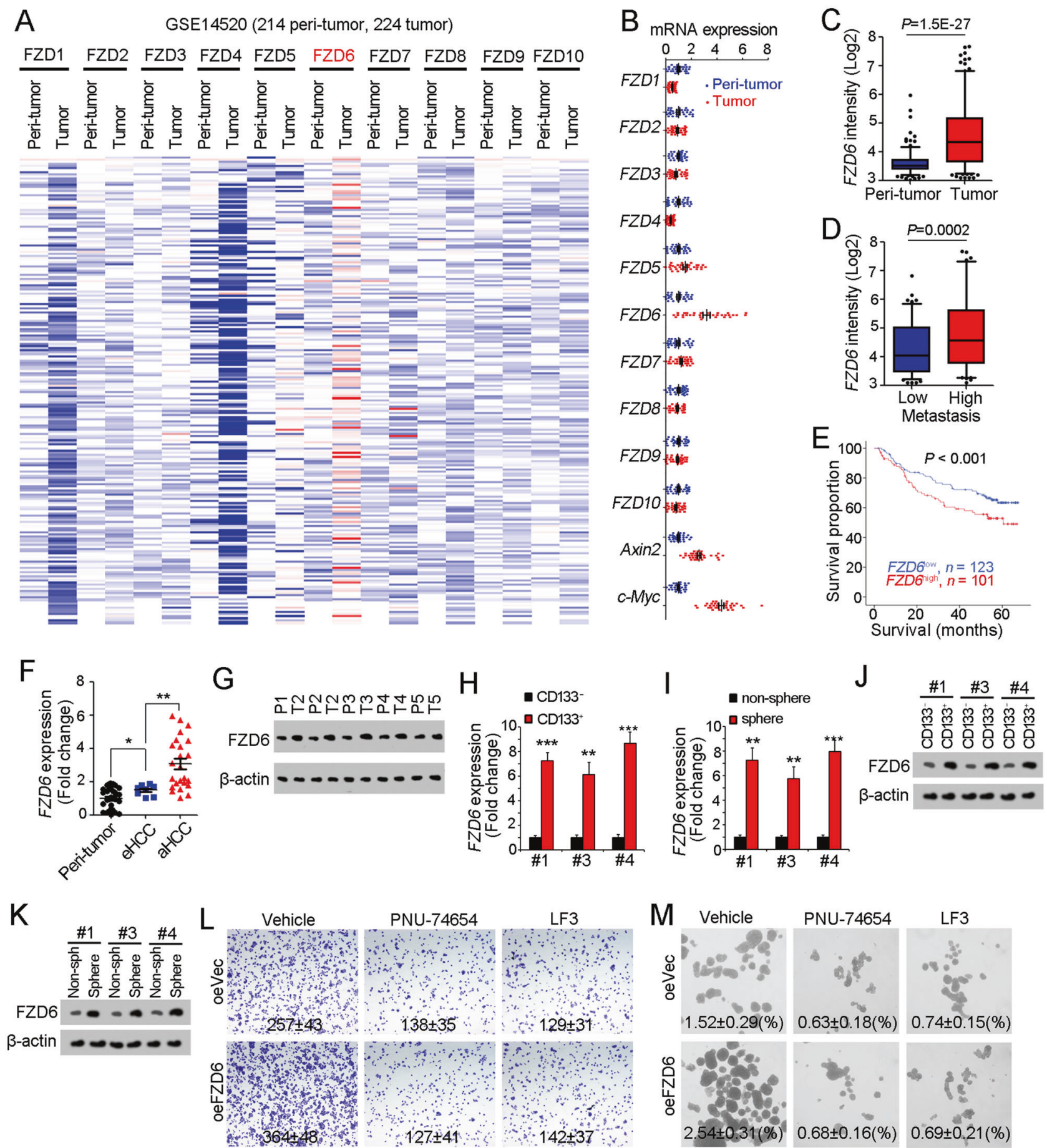

signaling and Hedgehog signaling [13-15]. Among these pathways, the role of $\mathrm{Wnt} / \beta$-catenin signaling in liver TICs is widely explored [16]. Wnt/ $\beta$-catenin signaling plays a central role in liver TIC self-renewal, and its activation is under precise regulation [17-19]. In Wnt-OFF cells, $\beta$ catenin interacts with APC, Axin2, $\beta$-TrCP to form APC complex, which triggered $\beta$-catenin ubiquitinoylation and subsequent degradation [20]. Upon Wnt ligands bind to their receptors (FZD1-FZD10), Wnt/ $\beta$-catenin signaling is activated. The conformational changes of FZD receptors induce the disruption of APC complex, releasing $\beta$-catenin from degradation complex, followed by nuclear translocation of $\beta$-catenin [21]. $\beta$-Catenin interacts with T-cell factor/ lymphoid enhancer-binding factor (TCF/LEF) complex in cell nuclei to drive target gene expression [22]. Although the regulatory mechanisms of $\mathrm{Wnt} / \beta$-catenin pathway have 
been deeply explored, how it is activated in liver tumorigenesis and liver TICs is largely unknown.

FZD receptors, encoded by "frizzled" gene family, are the receptors for Wnt molecules, and responsible for Wnt/ $\beta$ catenin activation upon Wnt binding [23]. There are 10 FZD receptors in human cells, FZD1-FZD10. Here we found FZD6 is highly expressed in liver cancer and liver TICs. FZD6 contains a signal peptide and a cysteine-rich domain in N-terminal and seven transmembrane domains [24]. However, unlike other FZD proteins, FCD6 does not contain a PDZ domain-binding motif in C-terminal [25]. The function of FZD6 in Wnt/beta-catenin activation is controversial. FZD6 acts as a negative regulator of Wnt3a induced $\mathrm{Wnt} / \beta$-catenin activation when co-expressed with FZD1 [26]. However, Wnt4 can bind to FZD6 and induce the activation of $\mathrm{Wnt} / \beta$-catenin/LEF/TCF signaling cascades, through a yet unknown mechanism [27]. The physiological and pathological role of FZD6 in Wnt//-catenin activation is still unclear.

Long noncoding RNAs (LncRNAs) are RNA transcripts with longer than 200 nucleotides in length and no proteincoding potential [28]. Recent studies reveals lncRNAs are important mediators in many biological processes, including tumorigenesis [29]. In tumors, IncRNAs participate in tumor initiation, metastasis, colony formation, energy metabolism and so on [30-33]. LncRNAs exert their roles through transcriptional regulation or post-transcriptional regulation. LncRNAs interact with chromatin remodeling complex and participate the transcription of neighbor or distant genes in cis or in trans [34]. For post-transcriptional regulation, IncRNAs combine with core components of signaling pathways, and change the stability or activity of associated proteins [29, 31]. Here, we found $\operatorname{lncFZD6}$ and FZD6 are highly expressed in liver cancer cells and liver TICs. LncFZD6 and FZD6 are required for liver TIC selfrenewal. LncFZD6 interacts and recruits BRG1 to FZD6 promoter to initiate transcription. LncFZD6-BRG1FZD6 signaling pathway can be a target for liver TIC elimination.

\section{Results}

\section{FZD6 is highly expressed in liver cancer and liver TICs}

Liver TICs account for liver initiation, invasion, metastasis and relapse. The self-renewal capacity of liver TIC selfrenewal is under precise regulation. Using unbiased screening for lncRNAs, we found that a lncRNA termed IncSox4 participated in liver TIC self-renewal and liver tumorigenesis. LncSox4 recruits Stat3 to the promoter of Sox4, and thus drives Sox4 expression and liver TIC self-
Fig. 1 High expression of FZD6 in liver cancer and liver TICs. a Heatmap of Wnt receptors (FZD1-FZD10) in 214 peri-tumor samples and 224 tumor samples derived from GSE14520. The average expression levels in peri-tumor samples were defined as 1. FZD6 is highly expressed during liver tumorigenesis. b Thirty-two peri-tumor samples and $32 \mathrm{HCC}$ samples were examined for expression levels of the indicated genes. Ten FZD members and two Wnt/ $\beta$-catenin target genes (Axin2, c-Myc) were analyzed. c, $\mathbf{d}$ Samples were divided into two groups according to sample origin $\mathbf{c}$ or metastasis d, and FZD6 intensity were analyzed and shown as box and whisker plot. Boxes indicate interquartile ranges (IQR); upper and lower edges are the 75th and 25th percentiles. Horizontal lines within boxes are median intensity. Whiskers are 5th and 95th percentiles. e Samples were divided into two groups according to FZD6 expression levels, followed by Kaplan-Meier survival analysis. f, g Peri-tumor, early hepatocellular carcinoma (eHCC) and advanced hepatocellular carcinoma (aHCC) samples were collected, and FZD6 expression levels were detected using real-time PCR $\mathbf{f}$ and western blot $\mathbf{g}$. h, i FZD6 mRNA expression levels were detected using CD133 enriched liver TICs $\mathbf{h}$ or oncospheres i. FZD6 expression in non-TICs was served as control. j, k liver TICs $\mathbf{j}$ and oncospheres $\mathbf{k}$ were enriched and FZD6 protein levels were examined with western blot. 1, m FZD6 overexpressed (oeFZD6) and control (oeVec) cells were treated with $\beta$-catenin/TCF inhibitors (PNU-74654, LF3), and tumor invasion $\mathbf{I}$ and self-renewal capacities m were examined. Typical images and calculated numbers (or ratios) were shown. For $\mathbf{b}, \mathbf{f}, \mathbf{h}, \mathbf{i}$, data were shown as means \pm s.d. For $\mathbf{c}, \mathbf{d}$, data are shown as box and whisker plot. $* P<0.05$; $* * P<0.01$; $* * * P$ $<0.001$ by two-tailed Student's $t$-test. Data are representative of three independent experiments

renewal [12]. Wnt $/ \beta$-catenin signaling is the most important signaling in liver TIC self-renewal. However, the initiation of $\mathrm{Wnt} / \beta$-catenin signaling is largely unknown.

The activation of $\mathrm{Wnt} / \beta$-catenin needs the combination of Wnt ligands and Wnt receptors. The expression levels of Wnt receptors play a fundamental role in $\mathrm{Wnt} / \beta$-catenin activation. Here, we examined the expression levels of Wnt receptor (FZD1-FZD10) using online available dataset (GSE14520) [35, 36]. Of the then FZD receptors, FZD6 is highly expressed along with liver tumorigenesis (Fig. 1a). We confirmed the high expression of FZD6 and Wnt/Bcatenin target genes (Axin2 and c-Myc) along with liver tumorigenesis using clinical samples (Fig. 1b). FZD6 is also related to liver tumorigenesis, tumor metastasis and prognosis (Figs. 1c-e). To further examine the expression pattern of FZD6, we collected liver cancer samples, examined the expression levels of FZD6 with real-time PCR and western blot, and confirmed the increased FZD6 expression both at mRNA levels and protein levels (Figs. 1f, g).

Considering the critical role of $\mathrm{Wnt} / \beta$-catenin in liver TIC self-renewal, we then detect FZD6 expression levels in liver TICs. We enriched liver TICs through two strategies: sorting with surface marker CD133 and sphere formation assay. We found both in CD133+ liver TICs and oncospheres, FZD6 is highly expressed (Figs. 1h, i). We then confirmed the real-time PCR results with western blot, and also found FZD6 is highly expressed in liver TICs at protein levels (Figs. 1j, k). Considering the role of FZD6 in Wnt/ $\beta$ - 
A

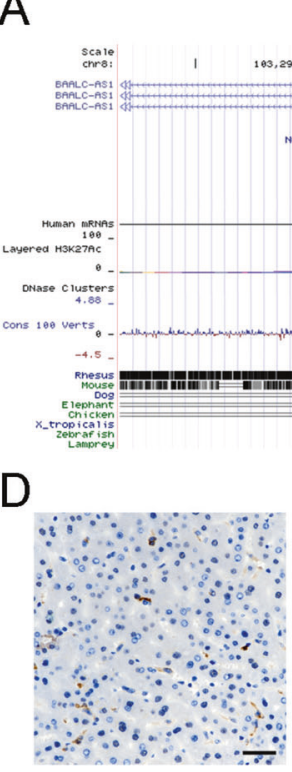

Peri-tumor

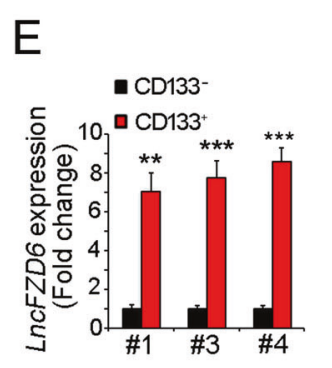

LnCFZD6

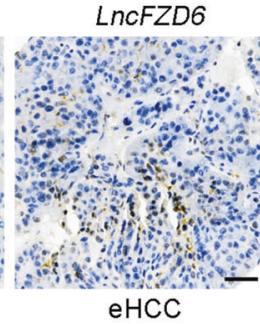

Human FZD6 locus

$\mathrm{F}$

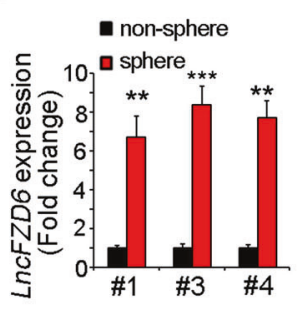

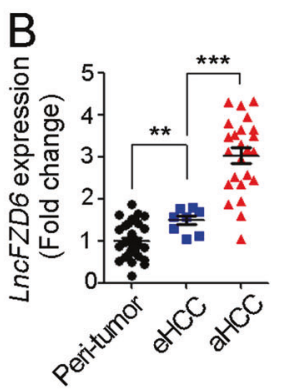

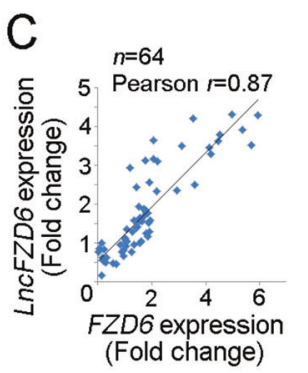

Fig. 2 LncFZD6 was highly expressed in liver cancer and liver TICs. a Human FZD6 locus was shown using UCSC Genome Browser. There is a divergent lncRNA (BAALC-AS1, here was termed as $l n c F Z D 6$ ) in near from FZD6 locus. b LncFZD6 expression levels were detected using real-time PCR. The average of lncFZD6 expression levels in peri-tumors were served as control. c The correlation between FZD6 and lncFZD6 expression levels. The fold changes of FZD6 and lncFZD6 in the sample tumor samples were used for scatter diagram, and Pearson correlation coefficient was calculated. Sixty-four samples were detected. $\mathbf{d}$ In situ hybridization (ISH) of $l n c F Z D 6$ in peri-tumor, early hepatocellular carcinoma (eHCC) and advanced hepatocellular

catenin activation is elusive, we overexpressed FZD6 in $\beta$ catenin inhibited cells, and found impaired influence on tumor invasion and self-renewal (Figs. 11, m), indicating that FZD6 drives liver tumor invasion and liver TIC selfrenewal through $\mathrm{Wnt} / \beta$-catenin signaling. Altogether, FZD6 is highly expressed in liver cancer and liver TICs.

\section{High expression of IncFZD6 in liver cancer and liver TICs}

We next wanted to explore the molecular mechanism of FZD6 expression. First, we silenced several TIC-related
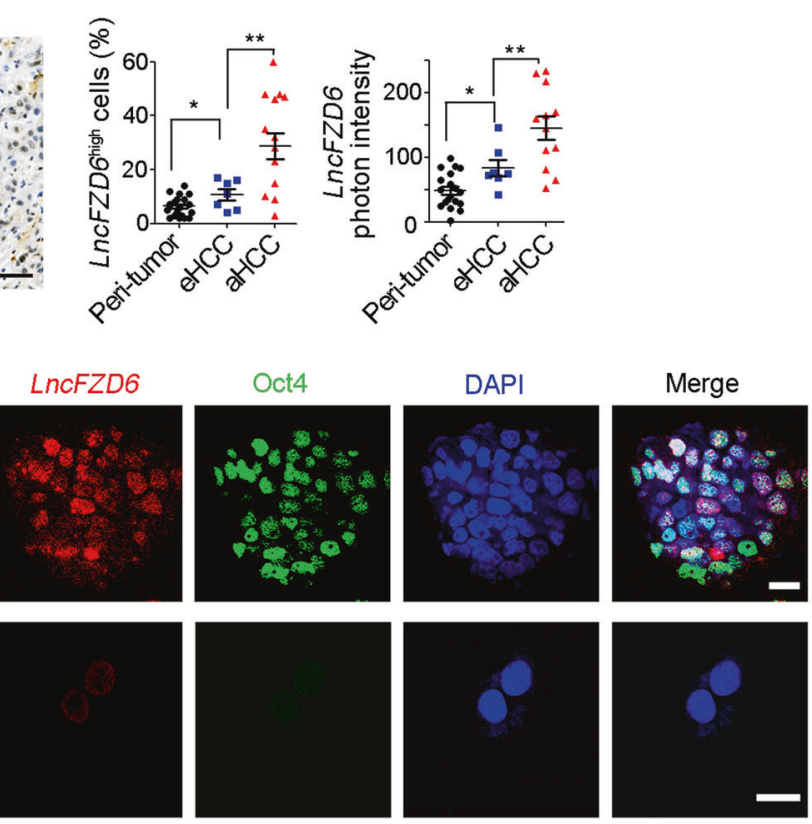

carcinoma (aHCC) samples. Typical photos were shown in left panels and calculated ratios were shown in right panels. e CD133 ${ }^{+}$liver TICs and CD133- liver non-TICs were enriched with FACS, followed by real-time PCR analysis for IncFZD6 expression. LncFZD6 expression levels in non-TICs were controls. Three samples were shown. f Sphere formation assays were performed, and lncFZD6 expression levels were detected with real-time PCR. g Fluorescence in situ hybridization of lncFZD6 using spheres and non-spheres. Oct4 was a positive control. Scale bars, $\mathbf{d}, 50 \mu \mathrm{m} ; \mathbf{g}, 10 \mu \mathrm{m}$. Data were shown as means \pm s.d. $* P<$ $0.05 ; * * P<0.01 ; * * * P<0.001$ by two-tailed Student's $t$-test. Data are representative of three independent experiments

molecules (including c-MYC, AXIN2, SOX2, TP53, STAT3, ZIC2, NOTCH2, SOX4, TCF7 and OCT4), and found knockdown of these genes had no changes of FZD6 mRNA expression, but FZD6 knockdown induced impaired c-MYC expression (Supplementary Figure 1A, B). These results indicated that FZD6 was a "upstream" factor and cannot be regulated by these key TIC-related molecules. To explore the mechanism of FZD6 expression, we focused on FZD6 gene locus, and found lncRNA ABBLC-ASC (hereafter termed lncFZD6) is a divergent lncRNA of FZD6. The distance of their transcription start sites is $<1 \mathrm{~kb}$ (Fig. 2a). LncFZD6 is also highly expressed in $\mathrm{HCC}$ 

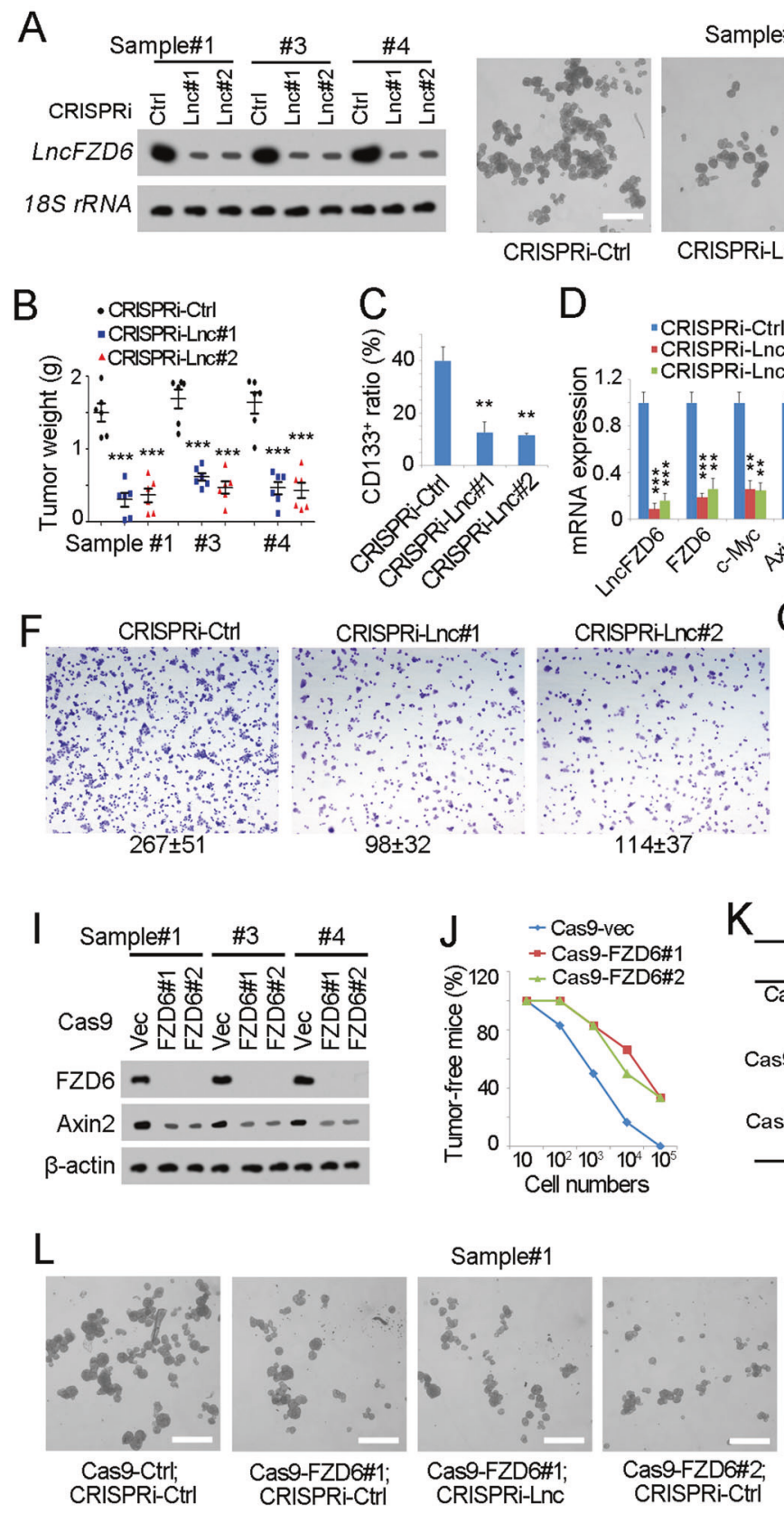

$\mathrm{M}$
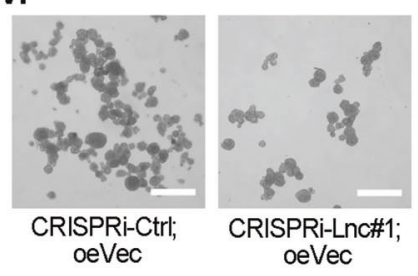

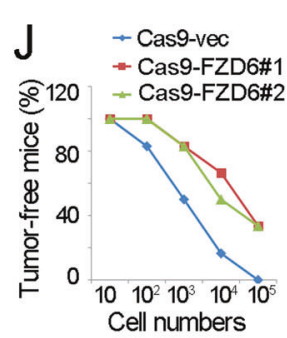

"CRISPRi-Ctrl
"CRISPRi-Lnc\#1

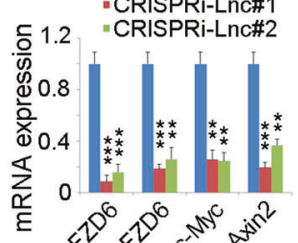

$\mathrm{K}$
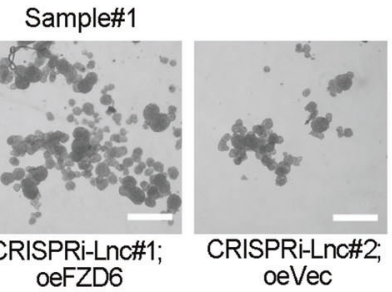

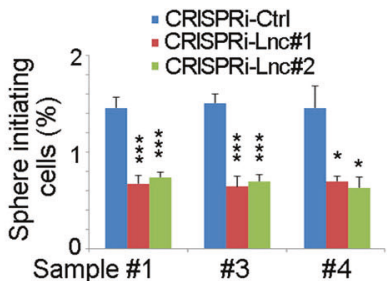

$\mathrm{E}$

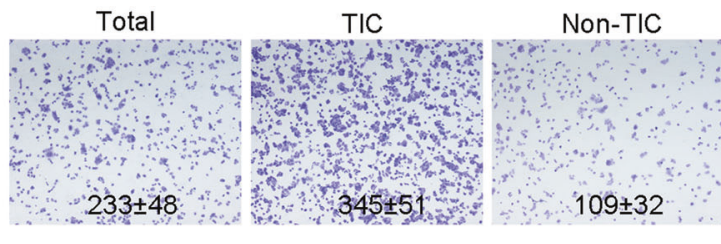

$\mathrm{H}$

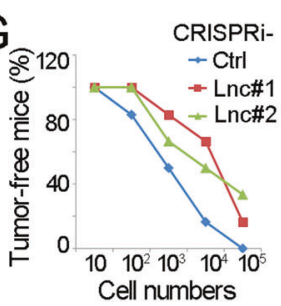

\begin{tabular}{ccc}
\hline Cell & TIC ratio $(95 \%$ Cl $)$ & p value \\
\hline $\begin{array}{c}\text { Cas9-Vec } \\
\text { (A) }\end{array}$ & $1 / 1 / 5791$ \\
& $(1 / 13812-1 / 2368)$ & \\
Cas9-FZD6\#1 & $1 / 57194$ & 0.0001 \\
(B) & $(1 / 138133-1 / 23681)$ & $(\mathrm{B}$ vs A) \\
Cas9-FZD6\#2 & $1 / 48257$ & 0.0003 \\
(C) & $(1 / 116278-1 / 20028)$ & $(\mathrm{C}$ vs A $)$ \\
\hline
\end{tabular}

samples, especially in advanced HCC samples (Fig. 2b). Interestingly, IncFZD6 is co-expressed with FZD6, with a high Pearson correlation coefficient (0.87) (Fig. 2c). We then detected IncFZD6 expression with in situ hybridization (ISH). The staining results showed increased lncFZD6
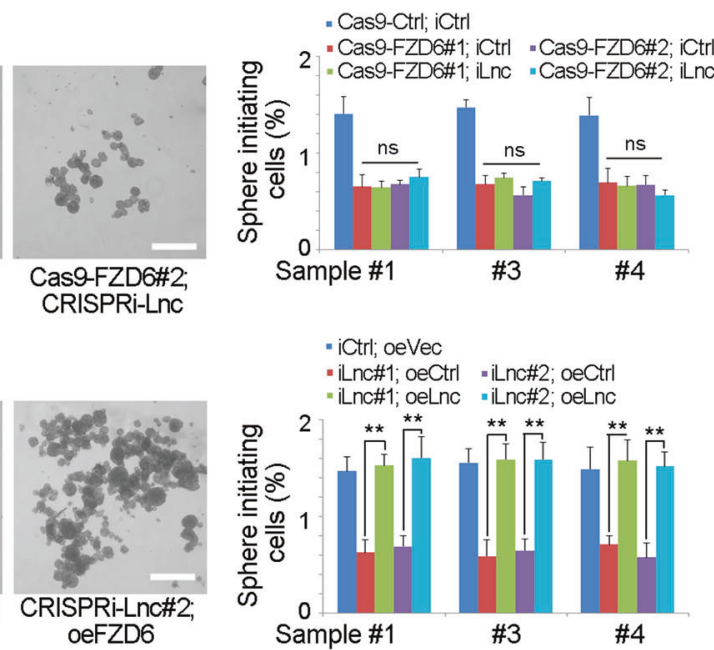

expression levels in liver tumor, especially in advanced liver tumor (Fig. 2d). Of note, only a small subset of tumor cells showed high expression of lncFZD6, even in advanced samples (Fig. 2d). 
Fig. 3 LncFZD6 was required for liver TIC self-renewal. a LncFZD6 silenced cells were established (left panels) with CRISPRi and sphere formation assays were performed. The efficiency of lncFZD6 knockdown was detected by northern blot (left panels). Typical pictures were shown in middle panels and calculated ratios were shown in right panels. b In all, $1 \times 10^{6} \operatorname{lncFZD6}$ silenced or control TICs were injected into $\mathrm{BALB} / \mathrm{c}$ nude mice. One month later, mice were sacrificed and the weight of tumors were detected and shown as scatter diagram. Six mice were used for each assay. $\mathbf{c}$ The indicated tumors were obtained and stained with CD133 antibody, followed by FACS examination. The ratios of $\mathrm{CD} 133^{+}$cells were shown. $n=6$ for each group. d Total RNA were extracted from the indicated tumors, and expression levels of the indicated transcripts were examined by realtime PCR. e CD133 ${ }^{+}$TICs and CD133- non-TICs were enriched and tumor invasion capacity was examined. Typical images and calculated ratios were shown. $\mathbf{f}$ lncFZD6 silenced or control cells were used for transwell assay, and typical images were shown. $\mathbf{g}, \mathbf{h}$ In all, 10, $1 \times$ $10^{2}, 1 \times 10^{3}, 1 \times 10^{4}$ and $1 \times 10^{5}$ lncFZD6 silenced cells and control cells were injected into BALB/c nude mice for 3 months' tumor formation. Three months later, tumor formation was observed and the ratios of tumor-free mice were calculated $\mathbf{g}$. TIC ratios were analyzed using extreme limiting dilution analysis h. 95\% CI 95\% confidence interval of the estimation, vs versus. i FZD6 knockout cells were generated with CRISPR/Cas9 approach, and knockout efficiency was determined with western blot. $\beta$-Actin served as a loading control. $\mathbf{j}, \mathbf{k}$ In all, $10,1 \times 10^{2}, 1 \times 10^{3}, 1 \times 10^{4}$ and $1 \times 10^{5}$ FZD6 knockout cells and control cells were injected into BALB/c nude mice and tumor formation was analyzed as $\mathbf{g}, \mathbf{h}$. $\mathbf{l}$ LncFZD6 was silenced in FZD6 knockout cells or control cells, followed by sphere formation assays. Typical pictures were shown in left panels and calculated sphereinitiating ratios were shown in right panels. m FZD6 expression was rescued in lncFZD6 depleted TICs, followed by oncosphere formation. Typical pictures were shown in left panels and TIC ratios were shown in right panels. Scale bars, $500 \mu \mathrm{m}$. Data were shown as means \pm s.d. $* P<0.05 ; * * P<0.01 ; * * * P<0.001$ by two-tailed Student's $t$-test; ns not significant. Data are representative of three independent experiments

As the high expression of FZD6 in liver TICs and the correlation between FZD6 and lncFZD6 expression pattern, we next examined the expression of lncFZD6 in liver TICs. We obtained $\mathrm{CD} 133^{+}$liver TICs and oncospheres, examined lncFZD6 expression, and found increased lncFZD6 transcripts in liver TICs (Figs. 2e, f). We then performed fluorescence in situ hybridization (FISH) with oncospheres and non-spheres, confirming the high expression levels of lncFZD6 in oncospheres (Fig. 2g). Altogether, lncFZD6 is highly expressed in liver cancer and liver TICs.

\section{LncFZD6 promotes the self-renewal of liver TIC}

We then explored the role of lncFZD6 in liver TIC selfrenewal. We depleted IncFZD6 expression with CRISPRi strategy, and found impaired sphere formation capacity upon $\operatorname{lncFZD6}$ depletion, indicating the critical role of lncFZD6 in liver TIC self-renewal (Fig. 3a). We also examined the role of lncFZD6 in liver TIC proliferation and apoptosis, and found lncFZD6 mainly affected the proliferation of liver TICs (Supplementary Figure 1C, D). We then injected $1 \times 10^{6}$ lncFZD6 silenced or control TICs into
$\mathrm{BALB} / \mathrm{c}$ nude mice, and found impaired tumor propagation of lncFZD6 depleted cells (Fig. 3b). What's more, decreased TIC ratios and impaired FZD6 were found upon lncFZD6 depletion, confirming the critical role of lncFZD6 in live TIC self-renewal and FZD6 expression (Figs. 3c, d). Using transwell assay, we also confirmed the enhanced invasion capacity of liver TICs and FZD6 drives liver tumor invasion (Figs. 3e, f). In vivo experiments also validated the critical of lncFZD6 in tumor invasion (Supplementary Figure 1E). To further detect the role of lncFZD6 in tumor initiation, we injected $10,1 \times 10^{2}, 1 \times 10^{3}, 1 \times 10^{4}$ and $1 \times$ $10^{5}$ lncFZD6 silenced cells into BALB/c nude mice, followed by 3 months' tumor formation. LncFZD6 silenced cells showed impaired tumor initiation capacities, confirming the critical role of IncFZD6 in liver TIC self-renewal (Figs. 3g, h).

We then wanted to know whether IncFZD6 participated in liver TIC self-renewal through FZD6. We established FZD6 knockout cells using CRISPR/Cas9 approach, and validated FZD6 knockout with western blot (Fig. 3i). FZD6 deleted cells showed impaired tumor initiation capacity (Figs. 3j, k), indicating the critical role of FZD6 in liver TICs. We then silenced lncFZD6 in FZD6 knockout cells, and found lncFZD6 knockdown had no obvious influence on liver TIC self-renewal, suggesting that lncFZD6 exert its role through FZD6-dependent manner (Fig. 31). FZD6 rescue in IncFZD6 depleted cells also rescued the impaired self-renewal capacity of liver TICs, confirming that lncFZD6 exerted its role through FZD6 (Fig. 3m). In conclusion, IncFZD6 drove liver TIC self-renewal through FZD6.

\section{LncFZD6 recruits BRG1 to FZD6 promoter}

We then explored the molecular mechanism of lncFZD6 in liver TIC self-renewal. We performed RNA pulldown assay and found a specific band in lncFZD6 enrichment, which was identified as BRG1 with mass spectrum, indicating the interaction between lncFZD6 and BRG1 (Fig. 4a). RNA pulldown and western blot also confirmed the interaction between IncFZD6 and BRG1 (Fig. 4b). We also constructed truncates and examined the interaction between these truncates and BRG1. The third region of lncFZD6 (\#3) was identified as the binding site with BRG1 (Fig. 4c). We also detected the physiological interaction between BRG1 and lncFZD6 using RNA immunoprecipitation (RIP) (Fig. 4d). Finally, we performed double FISH and found colocalization between lncFZD6 and BRG1 in oncospheres (Fig. 4e). Altogether, IncFZD6 interacts with BRG1 in liver TICs.

BRG1 is a core component of SWI/SNF complex and plays an essential role in chromatin remodeling and transcriptional regulation. So we next explored the role of lncFZD6 and BRG1 in FZD6 transcription regulation. First, 

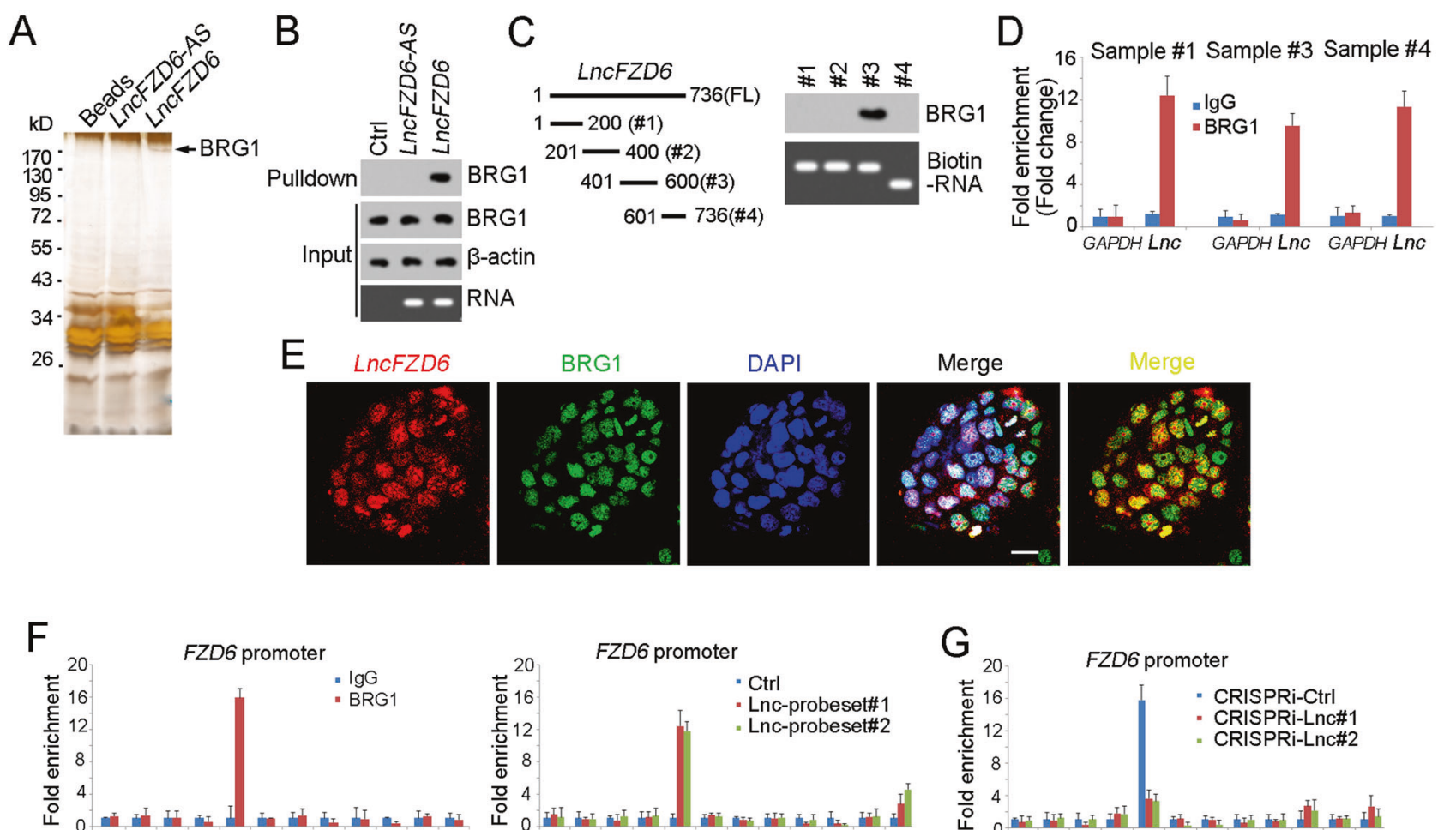

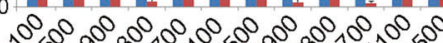

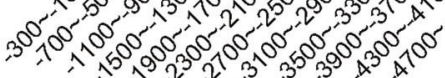

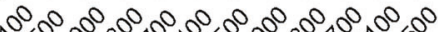

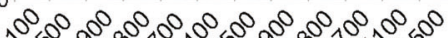
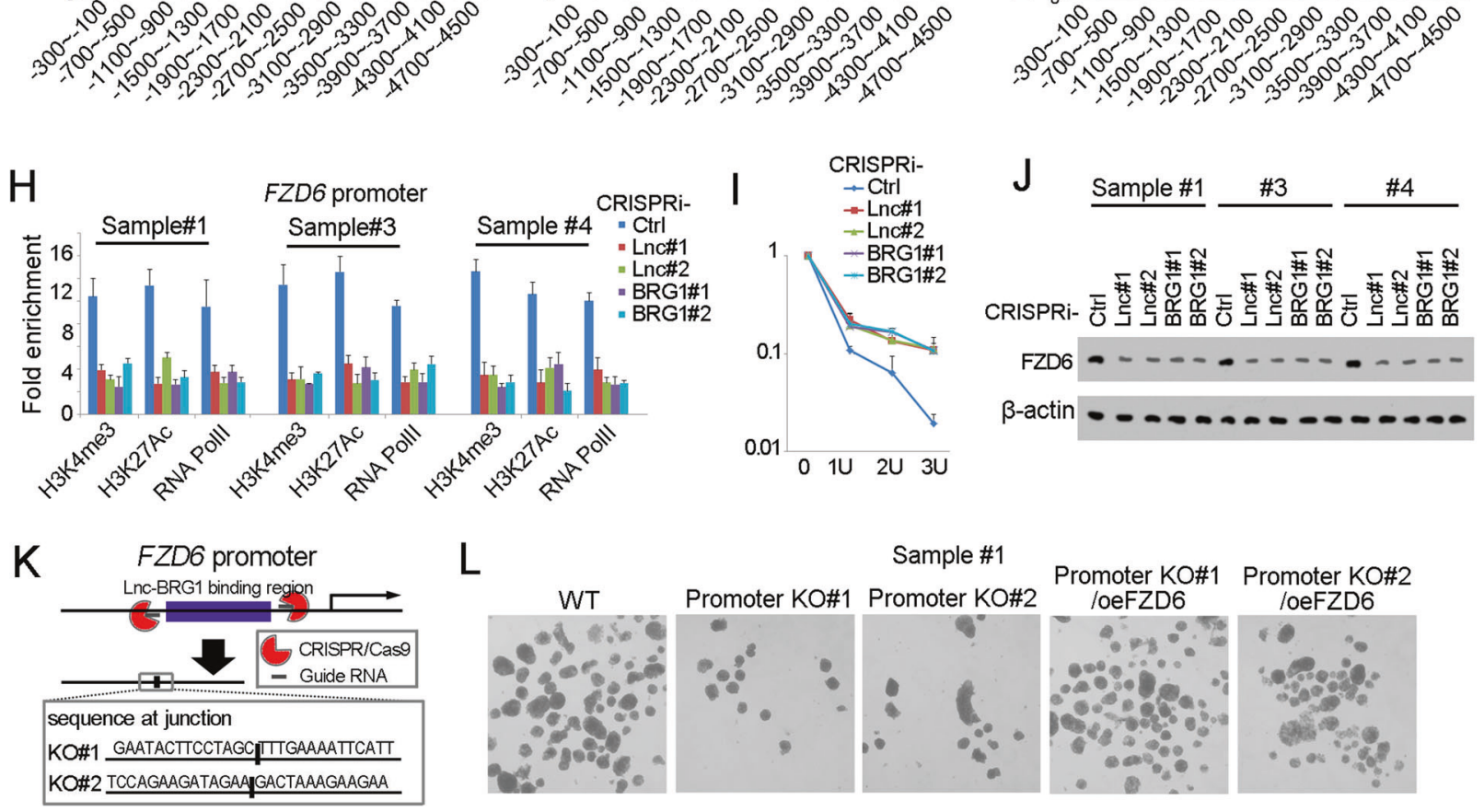

$\mathrm{L}$
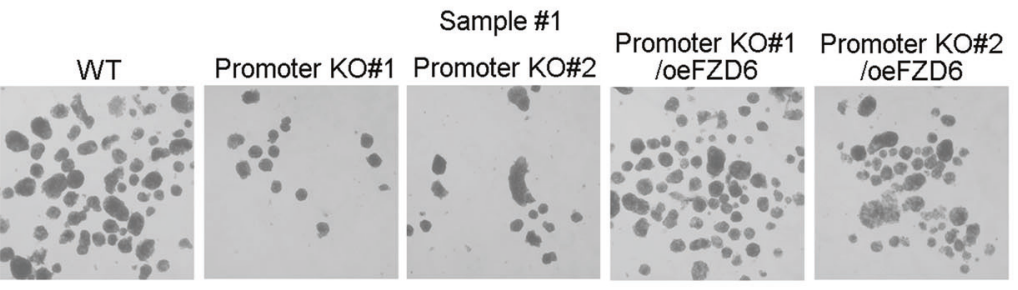

we performed chromosome immunoprecipitation (ChIP) and Chromatin isolation by RNA purification (ChIRP) assays, followed by FZD6 promoter examination, and found both BRG1 and IncFZD6 bound to FZD6 promoter. Of note, BRG1 and lncFZD6 interacted with the same region of FZD6 promoter (Fig. 4f). We then examined the interaction between BRG1 and FZD6 promoter in lncFZD6 silenced cells, and found impaired binding of BRG1 to FZD6 promoter upon lncFZD6 depletion, indicating the critical role of $\operatorname{lncFZD6}$ in the combination between BRG1 and FZD6 promoter (Fig. 4g).

We then detected the role of BRG1 and lncFZD6 in FZD6 transcriptional activities. Considering the critical role of BRG1 in histone modification, we detected the histone modification levels in lncFZD6 and BRG1 silenced cells, and found impaired histone modification upon $\operatorname{lncFZD6}$ and BRG1 depletion, indicating the involvement of lncFZD6 and BRG1 in FZD6 promoter activation (Fig. 4h). We also 
Fig. 4 LncFZD6 recruits BRG1 to FZD6 promoter. a RNA pulldown as performed and the specific band of lncFZD6 was identified as BRG1 with mass spectrum. b The interaction between lncFZD6 and BRG1 was confirmed by western blot. $\beta$-Actin served as a loading control. c LncFZD6 truncates were constructed (left panels), followed by RNA pulldown and western blot (right panels). d RNA immunoprecipitation (RIP) assay were performed with oncospheres derived from clinical samples, and enrichment of $\operatorname{lncFZD6}$ and GAPDH were examined with real-time PCR. IgG is an isotype antibody control. Data were shown as means \pm s.d. e Double FISH assays showed the colocalization of lncFZD6 and BRG1. Scale bars, $10 \mu \mathrm{m}$. f ChIP and ChIRP assays were performed with BRG1 antibody and lncFZD6 probes, respectively, followed by FZD6 promoter detection with realtime PCR. BRG1 and lncFZD6 bind to the same region of FZD6 promoter. g LncFZD6 silenced and control cells were used for BRG1 ChIP assay and impaired combination between BRG1 and FZD6 promoter was found in IncFZD6 silenced cells. h LncFZD6 and BRG1 silenced cells were used for ChIP assays with $\mathrm{H} 3 \mathrm{~K} 4 \mathrm{me} 3$, H3K27Ac and RNA polymerase II. FZD6 promoter enrichment was analyzed by real-time PCR. i DNase sensibility assays were performed using lncFZD6 and BRG1 silenced cells. j LncFZD6 and BRG1 silenced cells were lyzed for FZD6 western blot. $\beta$-Actin served as a loading control. $\mathbf{k}$ The $\operatorname{lncFZD6-BRG1}$ binding region on FZD6 promoter was deleted using CRISPR/Cas9 approach (upper panel), and confirmed by DNA sequencing (lower panel). I Sphere formation assays were performed using FZD6 promoter deleted cells (promoter KO) and FZD6 rescued cells (promoter KO/oeFZD6) cells, and typical images were shown. Data are representative of three independent experiments

performed DNase sensibility assay, and found enhanced resistance to DNase digestion, indicating the inactivation state of FZD6 promoter upon lncFZD6 and BRG1 depletion (Fig. 4i). Finally, we examined the expression levels of FZD6 with western blot using lncFZD6 and BRG1 silenced cells, and confirmed the positive regulation of FZD6 expression by lncFZD6 and BRG1 (Fig. 4j).

To further confirm the role of the combination between lncFZD6-BRG1 and FZD6 promoter, we deleted the lncFZD6-BRG1 binding region of FZD6 promoter (Fig. $4 \mathrm{k}$ ), and found impaired combination largely inhibited liver TIC self-renewal (Fig. 4l). Altogether, lncFZD6 interacted with BRG1 and recruited it to FZD6 promoter, and finally drove FZD6 expression.

\section{WNT5A is highly expressed in liver cancer cells}

We then detected FZD6 ligands in liver tumorigenesis. We analyzed WNT expression profiles using online available data, and found WNT5A is highly expressed in liver cancer (Fig. 5a). Using primary HCC samples, we confirmed the high expression of WNT5A in liver cancer, especially in advanced liver cancer (Figs. 5b-d).

We then examined transcriptional activation of WNT5A promoter in normal liver cells, peri-tumor cells, non-TICs and TICs, and found WNT5A promoter is specifically activated in non-TICs (Figs. 5e, f). High expression levels of WNT5A mRNA were also found in liver non-TICs (Fig. 5g).
We then observed WNT5A expression profiles in primary samples, and confirmed that WNT5A is expressed in CD133- or CD13- non-TIC cells (Fig. 5h).

We incubated oncospheres with recombinant WNT5A and found WNT5A binds to TIC surface (Fig. 5i). What is more, FZD6 knockout and FZD6 promoter knockout spheres showed impaired combination with WNT5A, indicating the essential role of FZD6 in liver TIC-WNT5A attachment (Fig. 5i). Accordingly, we added FZD6 antibody and found attenuate WNT5A binding, confirming FZD6-WNT5A interaction in liver TIC surface (Fig. 5i). Through Glutathione S transferase (GST) pulldown assay, we also confirmed the interaction between WNT5A and FZD6 (Fig. 5j). Altogether, WNT5A is the predominant ligand of FZD6 and mainly expressed in non-TICs.

\section{WNT5A drives Wnt/ $\beta$-catenin activation of TICs through IncFZD6-FZD6 signaling}

We then examined the role of WNT5A in liver TIC selfrenewal. First, we treated spheres with WNT5A and observed $\beta$-catenin activation by observing $\beta$-catenin subcellular location, and found WNT5A triggers $\beta$-catenin nuclear translocation and Wnt/ $\beta$-catenin activation (Fig. 6a). We then found WNT5A knockout spheres showed impaired self-renewal capacities, and recombinant WNT5A can rescue the diminished sphere formation (Fig. 6b). We also found the medium of WT spheres promote sphere formation of WNT5A knockout cells, but WNT5A knockout medium cannot, indicating WT sphere secret WNT5A to drive selfrenewal (Fig. 6c). What's more, non-TICs could promote the self-renewal of liver TICs and Wnt/ $\beta$-catenin activation, but WNT5A knockout non-TICs could not, indicating that non-TICs drove liver TIC self-renewal and Wnt/ $\beta$-catenin activation through WNT5A (Figs. 6d, e).

To further explore the role of WNT5A in liver cancer, we treated primary liver cancer cells with WNT5A, and found WNT5A treated cells showed enhanced Wnt/ $\beta$-catenin activation (Fig. 6f). What's more, WNT5A treatment had impaired roles in lncFZD6 silenced and FZD6 knockout cells, indicating lncFZD6-FZD6 axis plays an essential role in WNT5A-induced Wnt/ $\beta$-catenin activation (Fig. 6f). Expression profiles of $\mathrm{Wnt} / \beta$-catenin target genes confirmed the important role of lncFZD6 and FZD6 in Wnt/ $\beta$-catenin activation (Fig. 6g). Finally, we explored the role of WNT5A in liver TIC self-renewal using sphere formation assay, and found WNT5A treatment promotes liver TIC self-renewal, and lncFZD6-FZD6 plays an essential role in WNT5A-induced liver TIC self-renewal (Fig. 6h). Transwell assay also confirmed the critical role of lncFZD6FZD6 in WNT5A-induced tumor invasion (Fig. 6i). Altogether, WNT5A drives Wnt/ $\beta$-catenin activation through lncFZD6 and FZD6-dependent manner. 
A

A GSE14520 (214 peri-tumor, 224 tumor)

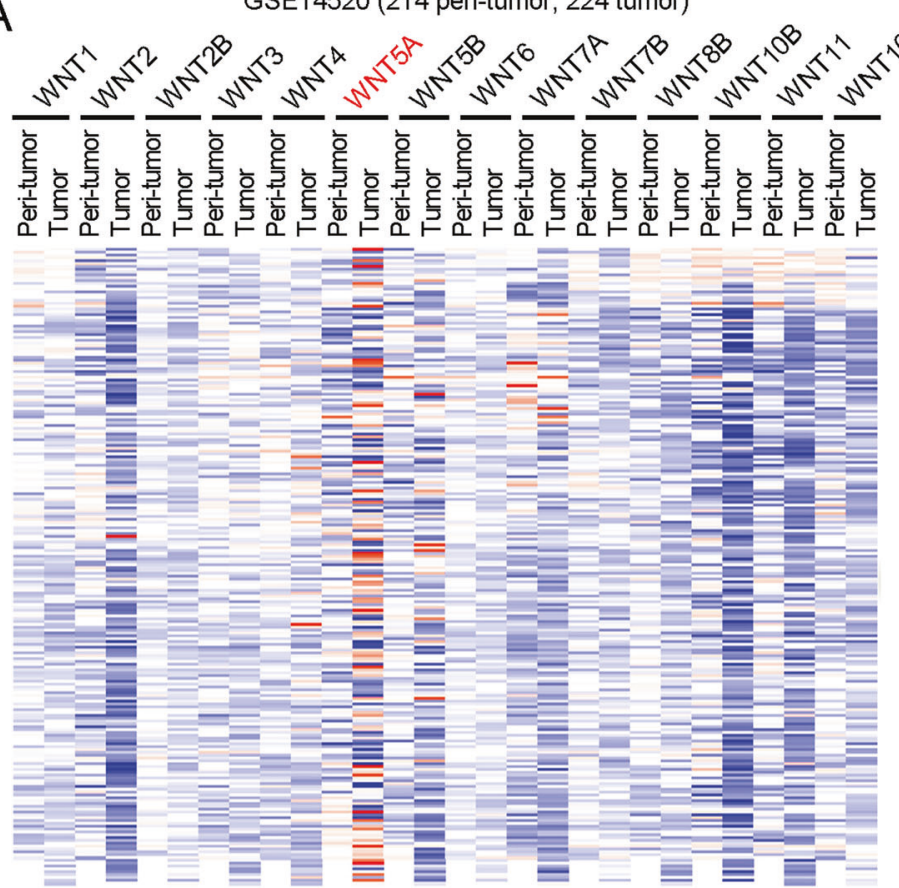

$B$

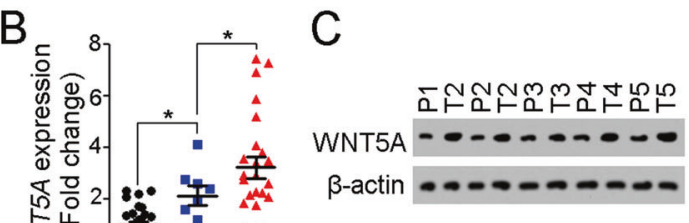

D
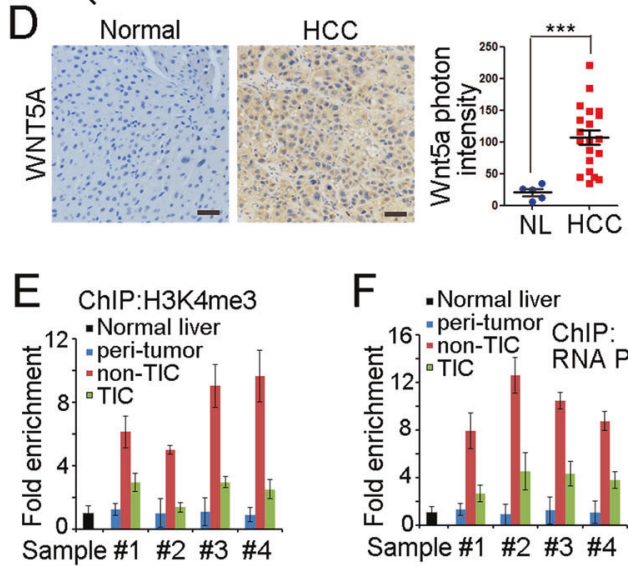

F . Normal liver

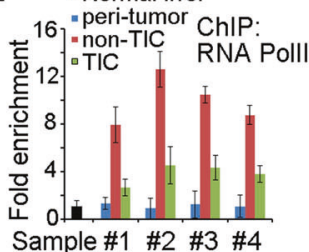

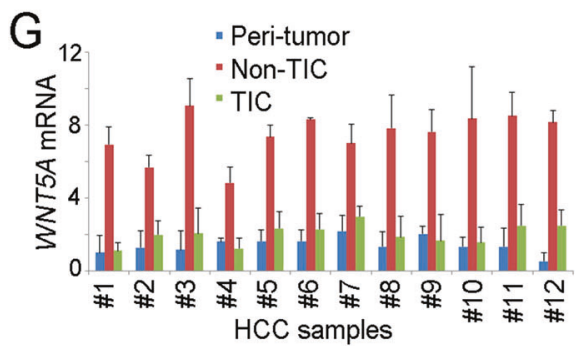

I

+ WNT5A

+ WNT5A+algG

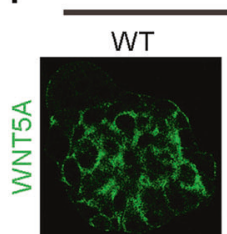
FZD6 KO Promot

FZD6KO/oe6
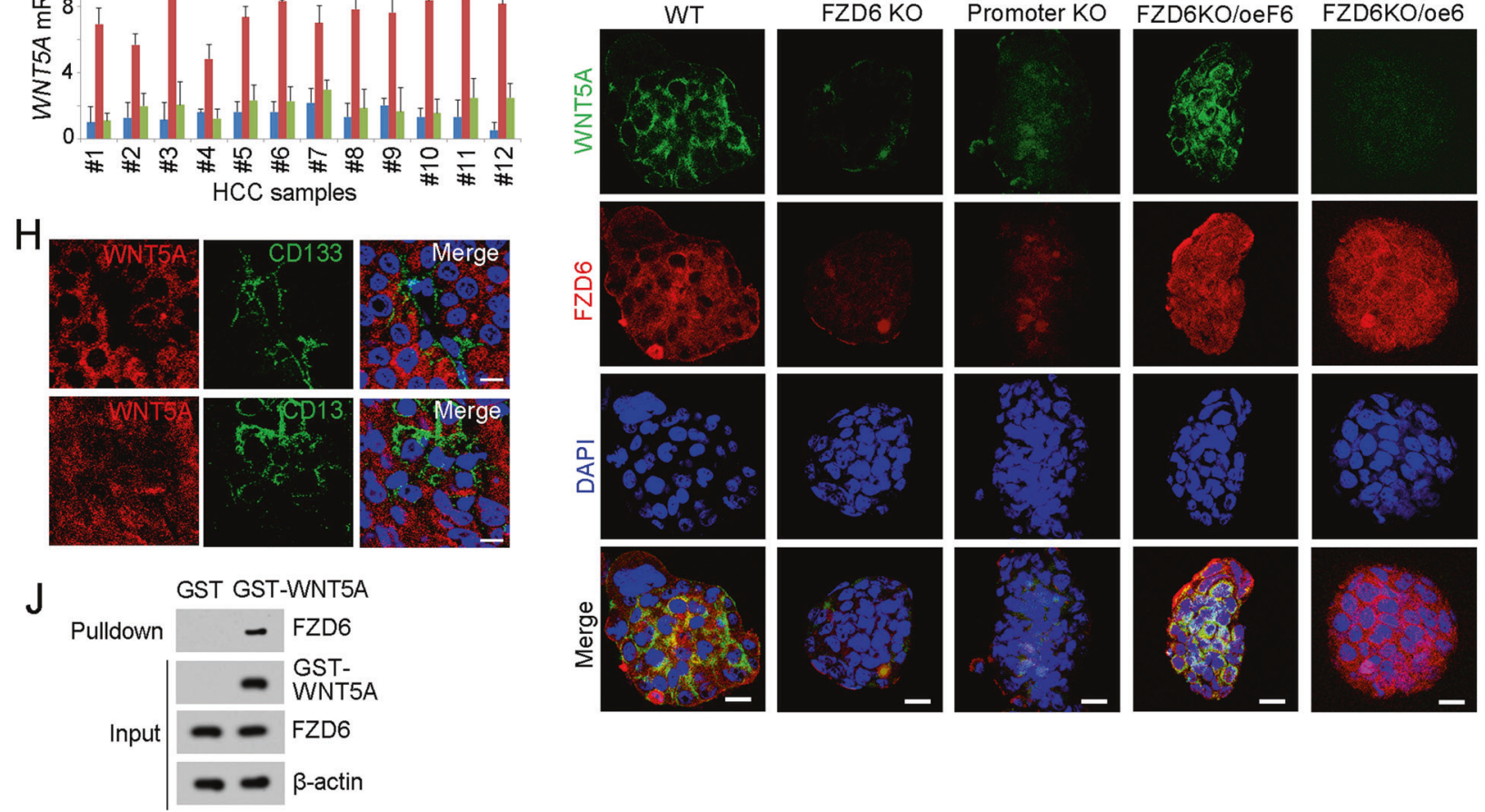

LncFZD6-BRG1-FZD6 serves as a target for liver TIC elimination

Finally, we explored the role of lncFZD6-BRG1-FZD6 in liver TIC elimination. We treated TICs with IncFZD6 ASO, FZD6 antagonist, BRG1 inhibitor PFI-3, or WNT5A inhibitor, and found impaired sphere formation upon inhibition of lncFZD6-BRG1-FZD6 pathway (Fig. 7a). We also found the critical role of lncFZD6-BRG1-FZD6 in liver tumor invasion (Fig. 7b). Taking advantage of tumor propagation assays, we also found lncFZD6-BRG1FZD6 pathway was required for liver TIC propagation 
Fig. 5 WNT5A is highly expressed in non-TICs. a Heatmap of indicated Wnt ligands in 214 peri-tumor samples and 224 tumor samples derived from GSE14520. The average expression levels in peri-tumor samples were defined as 1 . WNT5A is highly expressed during liver tumorigenesis. b, c Peri-tumor, early hepatocellular carcinoma (eHCC) and advanced hepatocellular carcinoma (aHCC) samples were collected, and FZD6 expression levels were detected using real-time PCR b and western blot c. d WNT5a expression levels in normal liver and HCC samples were examined with immunohistochemistry. Typical images were shown in left panels and calculated results were shown in right. e, f The activation of WNT5A promoter in the indicated cells were analyzed by ChIP assays with $\mathrm{H} 3 \mathrm{~K} 4 \mathrm{Me} 3$ and RNA polymerase II (RNA PolII). Normal liver cells, peri-tumor, non-TICs and TICs were sorted for ChIP assays. WNT5A is transcriptionally activated in normal liver tumor cells (non-TICs). $\mathbf{g}$ The indicated cells were enriched and WNT5A expression levels were analyzed using real-time PCR. h HCC primary samples were stained with WNT5A and CD133 (upper panel) or CD13 (lower panel), showing that WNT5A is highly expressed in CD133 and CD13 negative cells (non-TICs). Scale bars, $10 \mu \mathrm{m}$. $\mathbf{i}$ The indicted oncospheres were incubated with recombinant human WNT5A protein. After washing three times, the spheres were fixed with 4\%PFA and permeablized with Triton X-100 buffer. The samples were stained with WNT5A and FZD6 antibodies and observed with confocal OLYMPUS FV1200. Scale bars, $10 \mu \mathrm{m}$. j GST-WNT5A was incubated with liver TIC lysate, followed by GST pulldown and western blot. FZD6 antibody was used for FZD6WNT5A interaction. Data were shown as means \pm s.d. $* P<0.05$ by two-tailed Student's $t$-test; $* * * P<0.001$. Data are representative of three independent experiments

(Fig. 7c). Then, we collected the established tumors and examined liver TICs with functional marker Oct4 and surface marker CD133. We performed immunohistochemistry assay, and found impaired expression of Oct4 in lncFZD6-BRG1-FZD6 silenced cells, indicating their impaired self-renewal (Fig. 7d). We also examined liver TIC population with CD133, and found lncFZD6-BRG1FZD6 inhibition remarkably reduced the liver TIC numbers, again confirming that IncFZD6-BRG1-FZD6 can be used for liver TIC elimination (Fig. 7e). What's more, $\beta$ catenin nuclear translocation and $\mathrm{Wnt} / \beta$-catenin target gene (c-Myc) were impaired upon lncFZD6-BRG1-FZD6 blocking (Fig. 7f). Taken together, lncFZD6-BRG1-FZD6 blockade inhibits $\mathrm{Wnt} / \beta$-catenin activation and eliminates liver TICs.

\section{Discussion}

The self-renewal regulation of liver TICs is precisely regulated, and many signaling pathways are involved in liver TIC self-renewal. Wnt/ $\beta$-catenin signaling, the most important signaling in liver TICs, is mainly regulated by $\beta$ catenin stability and activity $[17,18]$. Upon Wnt activation, $\beta$-catenin is translocated into cell nuclei and activates the transcription of target genes [37]. The stability and transcriptional activity of $\beta$-catenin are most deeply explored; while, the initiation process of $\mathrm{Wnt} / \beta$-catenin activation is poorly understand. It is the interaction between Wnt and Wnt receptors that initiates $\mathrm{Wnt} / \beta$-catenin activation. Wnt molecules are secreted from "niche" cells, and Wnt receptors are highly expressed in TICs. There are many kinds of Wnt (Wnt1, Wnt2, Wnt3, Wnt3A, Wnt4, Wnt5A, Wnt5B, Wnt6, Wnt7A, Wnt7B, Wnt8A, Wnt8B, Wnt9A, Wnt10A, Wnt11, etc) and Wnt receptors (FZD1-FZD10). Here, we investigated the expression profile of Wnt receptors in tumorigenesis and liver TICs, and found FZD6 is highly expressed in liver cancer and liver TICs.

The role of FZD6 in $\mathrm{Wnt} / \beta$-catenin signaling activation is controversial. FZD6 blocks $\mathrm{Wnt} / \beta$-catenin activation by Wnt3a when co-expressed with FZD1 [26]. However, FZD6 is required for $\mathrm{Wnt} 4$ inducted $\mathrm{Wnt} / \beta$-catenin activation [27]. We think the opposite role of FZD6 in $\mathrm{Wnt} / \beta$ catenin activation maybe a result of different cell types and different Wnt ligands. As there is no PDZ domain-binding motif in C-terminal and the molecular mechanism of FZD6 in Wnt4 induced $\mathrm{Wnt} / \beta$-catenin activation remains unclear [27]. Here, we examined the role of FZD6 in liver TIC selfrenewal using FZD6 silenced and knockout cells, and found FZD6 is required for liver TIC self-renewal. What's more, FZD6 serves as a target for liver TIC elimination. Using various functional assays, we found FZD6 plays a positive role in liver tumorigenesis and TIC self-renewal, indicating its positive regulation of FZD6 in $\mathrm{Wnt} / \beta$-catenin activation along with tumorigenesis.

LncRNAs plays various roles in physiological and pathological processes [38]. Divergent lncRNAs, transcribed in the opposite direction to nearby protein-coding genes, are about $20 \%$ of total lncRNAs [39]. Divergent lncRNAs often co-expresses with its nearby genes, and regulate its nearby gene in cis [39]. Here we found lncFZD6 is a divergent IncRNA to FZD6 gene. LncFZD6 is coexpressed with FZD6 and regulate the expression of FZD6 through BRG1-mediated chromatin remodeling. There are several lncRNAs involved in $\mathrm{Wnt} / \beta$-catenin activation. A recent work showed lnc $\beta$-catm promoted the interaction between $\beta$-catenin and $\mathrm{EZH} 2$ to drive the methylation of $\beta$ catenin, and finally inhibited $\beta$-catenin ubiquitinoylation and activated Wnt/ $\beta$-catenin activation [40]. Here we found another lncRNA involved in Wnt receptor expression.

BRG1, a core component of SWI/SNF complex, plays critical role in tumorigenesis of many tumor types [41]. There are large amount of similarity $(>75 \%)$ of BRG1 and its analog, BRM. Both BRG1 and BRM can form SWI/SNF complex in exclusive manner [42]. Accordingly, SWI/SNF can be grouped to BRG1-embedded SWI/SNF complex and BRM-embedded SWI/SNF complex. Recently, a work discovered that BRG1-BRM switch occurred in liver tumorigenesis, and to a large extent, the switch accounted for liver TIC self-renewal [14]. Here we found BRG1 is 

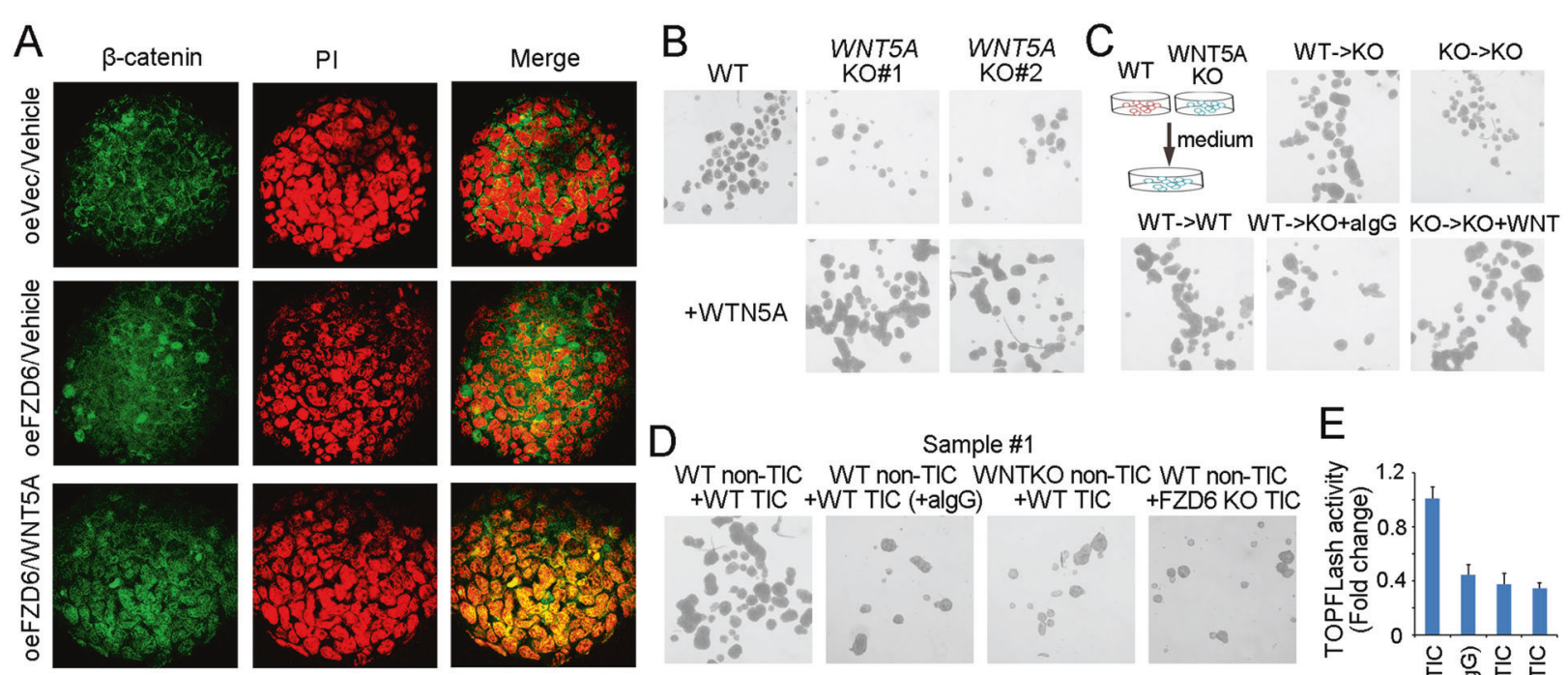

\section{E}

F
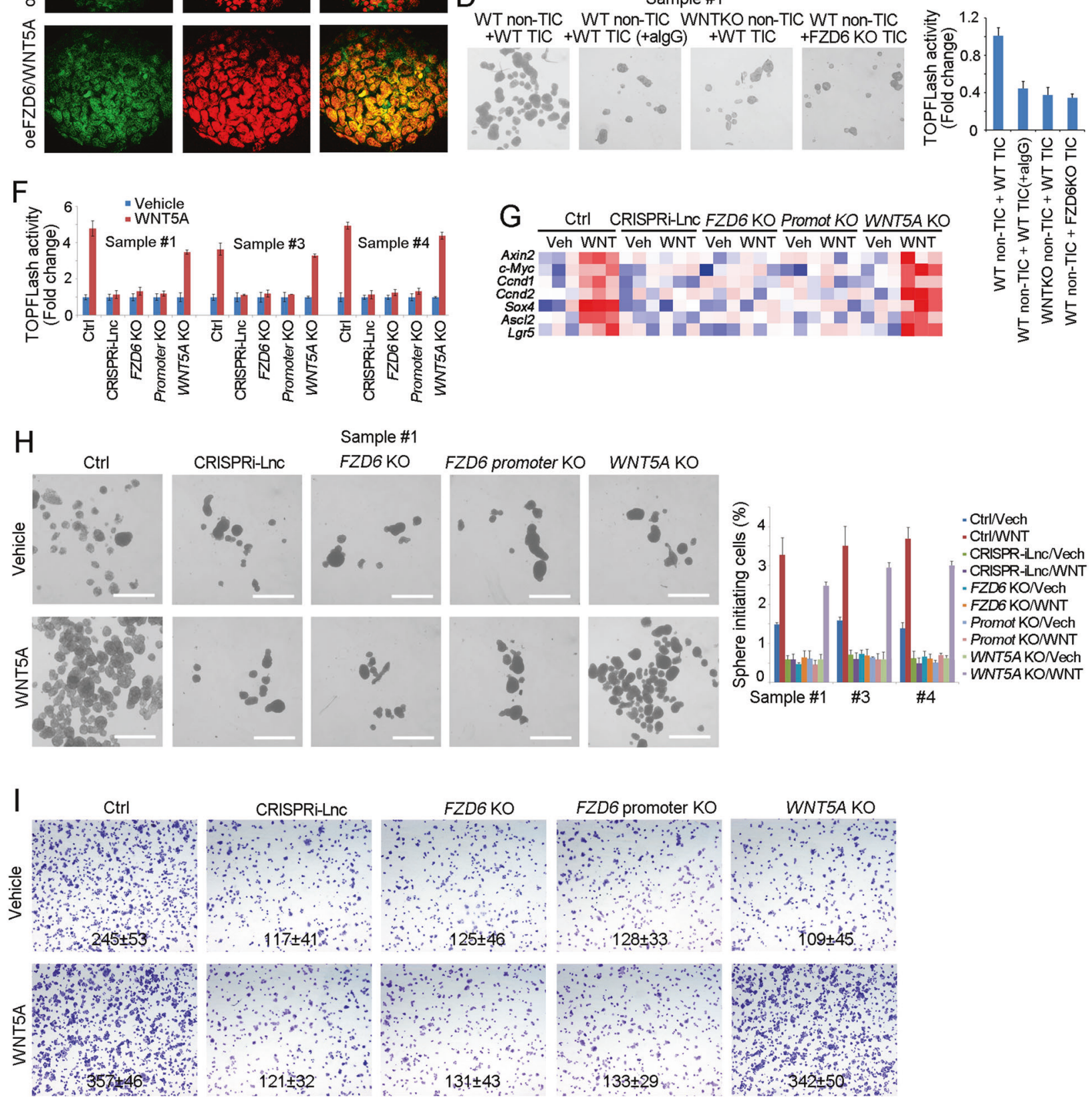

required for liver TIC self-renewal. BRG1 was recruited to FZD6 promoter by IncFZD6. LncFZD6-BRG1-FZD6 can be used for liver TIC elimination.
$\beta$-Catenin mutation has been discovered as a driver mutation in subpopulation of HCC patients in clinic, and here we found another driven gene. Thus, we compared $\beta$ - 
Fig. 6 WNT5A activates Wnt// -catenin signaling through FZD6dependent manner. a The indicated treated spheres were examined for $\beta$-catenin nuclear translocation by confocal microscope. b Sphere formation assays were performed using WNT5A knockout cells, supplemented with recombinant WNT5A protein or not. c WT and WNT5A knockout cells were used for 1 week's sphere formation and the supernatant medium were obtained for sphere formation of WNT5A knockout cells. Two weeks later, the typical spheres were shown. d, e WT and WNT5A KO non-TICs were sorted and mixed with TICs at ratio of $1: 1$, followed by oncosphere formation assay $\mathbf{d}$ or TOPFLash assay e. $\operatorname{aIgG}$, WNT5A antibody IgG. f The indicated cells were treated with WNT5A, and Wnt/ $\beta$-catenin activation was examined using TOPFLash methods. $\mathrm{g}$ The indicated cells were treated with WNT5A for 2 days, and the expression levels of Wnt/ $/$-catenin target genes were examined by real-time PCR. The expression data were shown as heatmap. h In all, 1000 indicated cells were incubated in sphere formation medium with/o WNT5A for 2 weeks. Typical pictures were shown in left panels and calculated sphere-initiating ratios were shown in right panels. Scale bars, $500 \mu \mathrm{m}$. i The indicated cells were incubated with WNT5A, and tumor invasion capacity was observed with transwell assay. Typical images and invasive cell numbers (mean \pm s.d.) were shown. Data were shown as means \pm s.d. ${ }^{*} P<0.05$ by two-tailed Student's $t$-test. Data are representative of three independent experiments

catenin mutation-driven HCC tumorigenesis and FZD6driven HCC tumorigenesis. We found no FZD6 expression changes in $\beta$-catenin mutant HCC samples (Supplementary Figure $1 \mathrm{~F}$ ), and $\beta$-catenin mutation (T41A) largely impaired the role of FZD6 (Supplementary Figure 1G, H). These results indicated that FZD6-initiated signaling was upstream of $\beta$-catenin and FZD6 functions through $\beta$-catenin signaling. The detailed crosstalk needs to be further explored.

\section{Materials and methods}

\section{Cells and samples}

293T cells (ATCC CRL-3216) and HCC cell line PLC (ATCC CRL-8024) were obtained from ATCC. Cells were cultured in Dulbecco's modified Eagle's medium (DMEM) medium (GIBCO), supplemented with $100 \mu \mathrm{g} / \mathrm{ml}$ penicillin, $100 \mathrm{U}$ streptomycin and $15 \%$ fetal bovine serum (FBS; Invitrogen).

Human liver cancer clinical samples were obtained from the first affiliated hospital of Zhengzhou University with informed consent, according to the Institutional Review Board approval. All human sample and mouse experiments were approved by the Institutional Committee of Zhengzhou University. HCC samples were ranked according to the obtained time, and several samples with sphere formation capacity (\#1, \#3 and \#4) were used for sphere formation. The details for these sample were: \#1, advanced HCC, 58 years old, male, tumor size, $7.8 \times 5.2 \times 4.9 \mathrm{~mm}$, nonmetastasis; \#3, advanced HCC, 71 years old, male, tumor size, $8.2 \times 4.3 \times 3.2 \mathrm{~mm}$, non-metastasis; \#4, advanced
HCC, 65 years old, female, tumor size, $5.8 \times 5.2 \times 4.6 \mathrm{~mm}$, non-metastasis.

\section{Antibodies and reagents}

DAPI (cat. no. 28718-90-3) and anti- $\beta$-actin antibody (cat. no. A1978) were purchased from Sigma-Aldrich. AntiFZD6 (cat. no. HPA017991) antibody was from Atlas antibodies, anti-RNA polymerase II (cat. no. GWB3F12B0) antibody was from GenWay. Anti-Oct4 (cat. no. 2750), anti-HeK4me3 (cat. no. 9727), anti-H3K27Ac (cat. no. 8173) antibodies were from Cell Signaling Technology. Anti-BRG1 (sc-17796) antibody was purchased from Santa Cruz Biotechnology. Phycoerythrin (PE)-conjugated CD133 (cat. no. 130098826) was from MiltenyiBiotec. Alexa594-conjugated donkey anti-rabbit $\mathrm{IgG}$ and Alexa488-conjugated donkey anti-mouse $\operatorname{IgG}$ antibodies were from Molecular Probes. T7 RNA polymerase (cat. no. 10881767001) and Biotin RNA Labeling Mix (cat. no. 11685597910) were obtained from Roche Life Science. The LightShift Chemiluminescent RNA EMSA kit (cat. no. 20158) and Chemiluminescent Nucleic Acid Detection Module (cat. no. 89880) were purchased from Thermo Scientific.

\section{Sphere formation}

For sphere formation assay, 5000 primary HCC cells were seeded into Ultra Low Attachment six-well plates and cultured in DMEM/F12 medium supplemented with N2, B27, $20 \mathrm{ng} / \mathrm{ml}$ EGF and $20 \mathrm{ng} / \mathrm{ml}$ bFGF for 2 weeks' incubation. The materials needed for sphere formation were: ultra low attachment plates (Corning, cat. no. 3471); N2 supplement (Life Technologies, cat. no. 17502-048), B27 (Life Technologies, cat. no. 17504-044), epidermal growth factor (EGF) (Life Technologies,cat. no. E5036-200UG) and basic fibroblast growth factor (bFGF) (Millipore, cat. no. GF44650UG).

\section{Real-time PCR}

Tissue RNA was extracted by TRIZOL method according to the Life's manual. Briefly, $1 \mathrm{ml}$ TriZol reagent was added into samples for 5-min incubation, followed by addition of $200 \mu \mathrm{l}$ chloroform and then the samples were separated by centrifugation. The supernatant was incubated with isopropanol and washed with $75 \%$ ethanol. Finally, the RNA samples were dissolved with RNase-free $\mathrm{H}_{2} \mathrm{O}$ and used as template for reverse transcription-PCR (RT-PCR). RT-PCR was performed with RT-PCR kit derived from Promega Company, and real-time PCR was performed by standard procedures. 
A

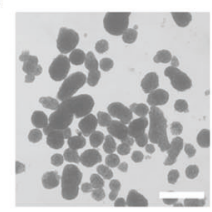

Vehicle

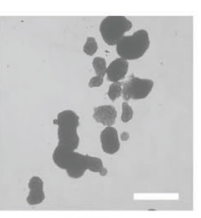

ASO
Sample \#3

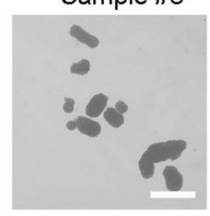

PFI-3

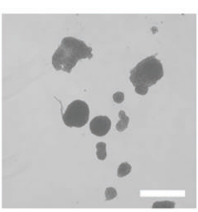

SFRP1

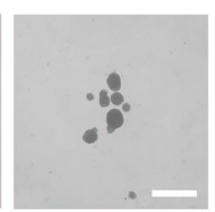

Box5

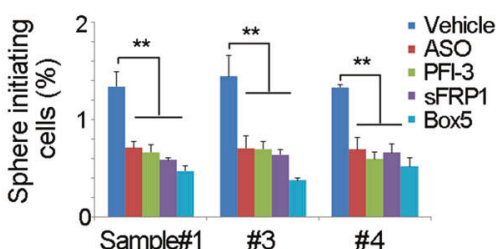

B

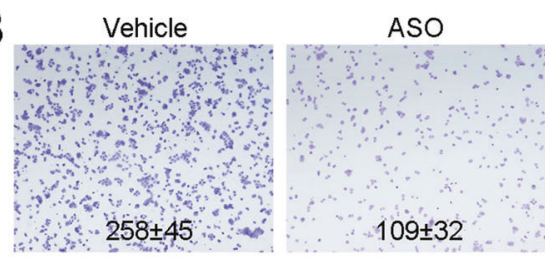

C

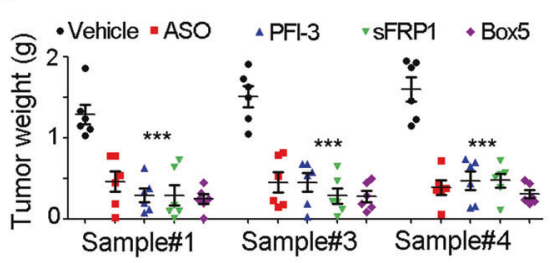

D
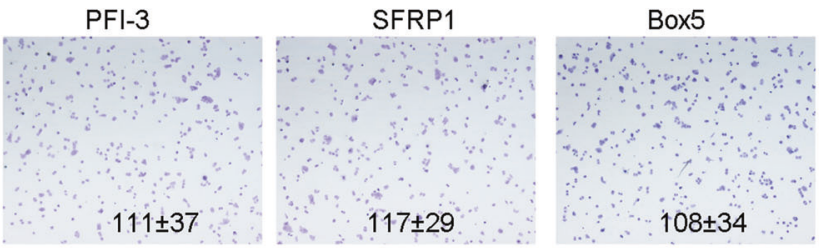

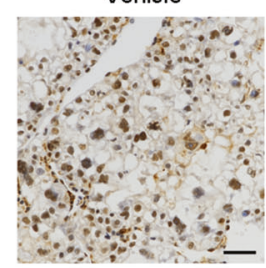

ASO

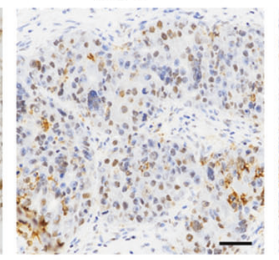

$\mathrm{PFI} 3$

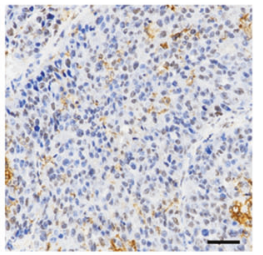

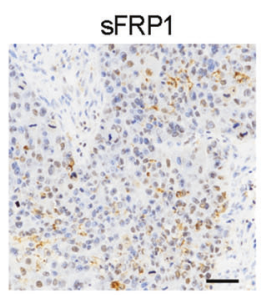

Box5

E。
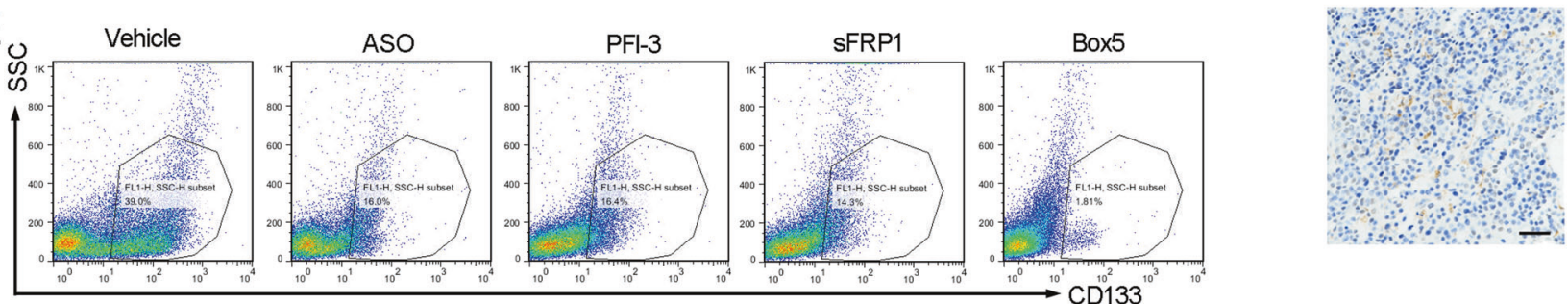

$\mathrm{F}$
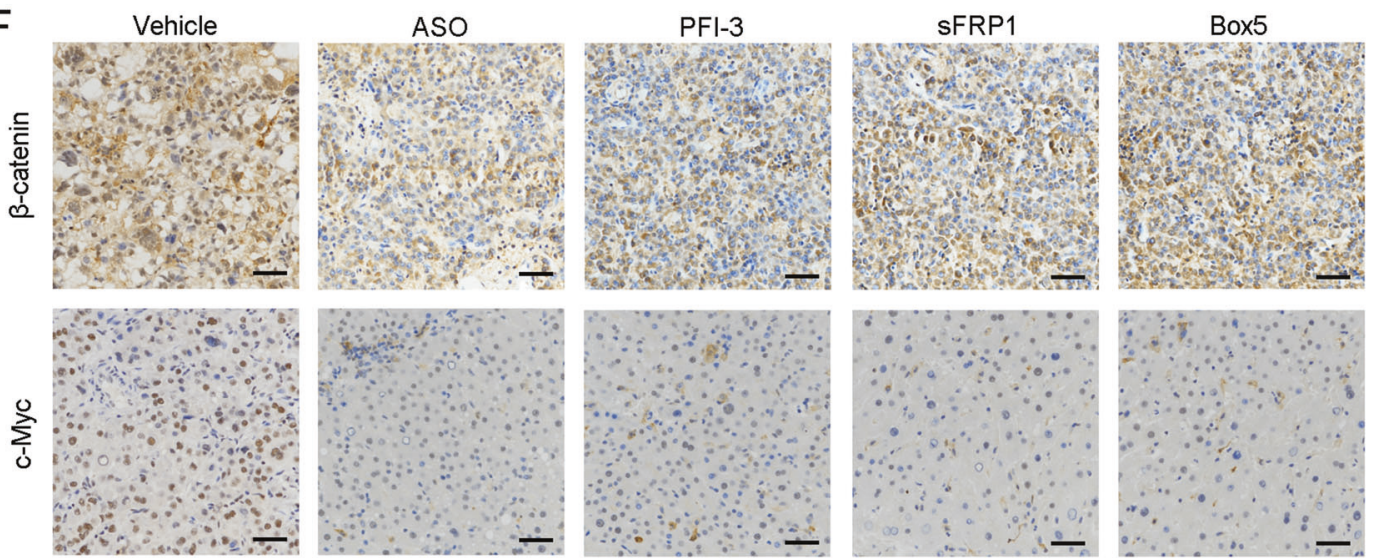

Fig. 7 LncFZD6-BRG1-FZD6 can be targeted for liver TIC elimination. a The indicated treated cells were used for sphere formation assays. Typical images were shown in left panels and calculated ratios were shown in right panels. b Tumor invasion capacity of the indicated treated cells were examined by transwell assay, and typical images and cell numbers (mean \pm s.d.) were shown. c In all, $1 \times 10^{6} \operatorname{lncFZD6}$ silenced (ASO), BRG1 inhibited (PFI-3), FZD6 antagonist (sFRP1) and WNT5A inhibitor (Box5) treated cells were injected into BALB/c nude mice for 1 month's tumor propagation. Tumor weights were shown as scatter diagram. Six mice were used for each assay. d The

indicated treated tumors were obtained and immunohistochemical analysis for the expression of Oct4, a functional marker of liver TICs. e CD133 FACS results of the indicated treated tumors. CD133 ${ }^{+}$liver TICs were gated and the proportion of liver TICs in tumor bulk were shown. $f$ The indicated treated tumors were obtained and immunohistochemical analysis for the expression of $\beta$-catenin and c-Myc. Scale bars, a, $500 \mu \mathrm{m} ; \mathbf{d}, \mathbf{f}, 50 \mu \mathrm{m}$. For a, c, data were shown as means \pm s.d. $* * P<0.01 ; * * * P<0.001$ by two-tailed Student's $t$-test; ns not significant. Data are representative of four independent experiments 


\section{Western blot}

HCC samples or oncospheres were crushed within RIPA buffer $(150 \mathrm{mM} \mathrm{NaCl}, 0.5 \%$ sodium deoxycholate, $0.1 \%$ sodium dodecyl sulfate (SDS), $1 \mathrm{mM}$ EDTA, $1 \%$ NP-40 and $50 \mathrm{mM}$ Tris, $\mathrm{pH}$ 8.0), followed by SDS-polyacrylamide gel electrophoresis (PAGE) for separation. The samples in the polyacrylamide gel were transferred to nitrocellulose (NC) membrane (Beyotime Biotechnology), and then incubated with primary antibodies and horseradish peroxidase (HRP)-conjugated secondary antibodies.

\section{Immunohistochemistry}

In all, 5- $\mu \mathrm{m}$ formalin-fixed sections were deparaffinized and rehydrated with xylene and graded alcohols. After 15-min incubation with $3 \%$ hydrogen peroxide $\left(\mathrm{H}_{2} \mathrm{O}_{2}\right)$, the slides were boiled in Tris-EDTA buffer $(10 \mathrm{mM}, \mathrm{pH} 8.0)$ for antigen retrieval. Then, the samples were incubated in primary antibodies and HRP-conjugated secondary antibodies. After detection with HRP substrate, the samples were counterstained with hematoxylin, followed by dehydration in graded alcohols and xylene.

\section{Tumor propagation and initiating assay}

For tumor propagation detection, $1 \times 10^{6}$ lncFZD6 silenced, overexpressed and control cells were injected 6-week-old $\mathrm{BALB} / \mathrm{c}$ nude. The mice were sacrificed 1 month later, and tumor weight was detected. For tumor-initiating assays, 10 , $1 \times 10^{2}, 1 \times 10^{3}, 1 \times 10^{4}$, and $1 \times 10^{5}$ lncFZD6 silenced cells were injected 6-week-old BALB/c nude for 3 months' tumor formation. The ratios of tumor-free mice were calculated 3 months later. For each sample of tumor propagation and tumor initiation, six mice were used.

\section{Transwell invasion assay}

For transwell invasion assays, $3 \times 10^{5} \mathrm{HCC}$ cells were plated on the top chamber with Matrigel-coated membrane, and incubated in medium without FBS. Medium supplemented with FBS was added in the lower chamber as a chemoattractant. The plate was incubated in incubator for $36 \mathrm{~h}$ and cells that did invade through the membrane were removed by a cotton swab. Cells on the lower surface of the membrane were fixed with methanol and stained with crystal violet. The images were taken with Nikon-EclipseTi microscopy.

\section{Chromosome immunoprecipitation}

ChIP assays were performed according to the manual of Upstate Biotechnology. Briefly, IncFZD6 silenced spheres or wide type spheres were incubated in $1 \%$ formaldehyde at
$37{ }^{\circ} \mathrm{C}$ for crosslinking, followed by treatment with SDS lysis buffer and subsequent ultrasonic for shearing DNA. BRG1, H3K4Me3, H3K27Ac and RNA polymerase II and control antibodies was added into samples for DNA segment enrichment.

\section{RNA pulldown}

For RNA pulldown assays, biotin-labeled $\operatorname{lncFZD6}$ and control RNA were prepared in vitro with biotin RNA labeling mix (Roche). Then, the labeled RNA transcript was incubated with sphere lysate. Streptavidin beads were added and the enriched components were analyzed by SDS-PAGE and western blot or mass spectra.

\section{RNA immunoprecipitation}

For RIP, liver TIC oncospheres were treated with $1 \%$ formaldehyde for crosslinking, and then lyzed with RNase-free RIPA buffer supplemented. The supernatants were incubated with BRG1 or control IgG antibodies and total RNA was extracted from the eluent. LncFZD6 or control ACTB enrichment was detected using real-time PCR.

\section{Statistical analysis}

Two-tailed Student's $t$-tests were used for statistical analysis. $P<0.05$ was considered to be statistically significant.

Acknowledgements This work was supported by the National Natural Science Foundation of China (U1704174, U1604286, 81472557 and 81601450 ) and development fund for outstanding young teachers of Zhengzhou University (1521311059).

Author contributions $\mathrm{ZC}$ and $\mathrm{YG}$ performed experiments, analyzed data and wrote the paper; LY, YL and ZY performed experiments and analyzed data; LH provided HCC samples and analyzed data; PZ and HW designed experiments and analyzed data; $\mathrm{ZC}$ initiated the study, designed experiments and wrote the paper.

\section{Compliance with ethical standards}

Conflict of interest The authors declare that they have no conflict of interest.

Open Access This article is licensed under a Creative Commons Attribution 4.0 International License, which permits use, sharing, adaptation, distribution and reproduction in any medium or format, as long as you give appropriate credit to the original author(s) and the source, provide a link to the Creative Commons license, and indicate if changes were made. The images or other third party material in this article are included in the article's Creative Commons license, unless indicated otherwise in a credit line to the material. If material is not included in the article's Creative Commons license and your intended use is not permitted by statutory regulation or exceeds the permitted use, you will need to obtain permission directly from the copyright holder. To view a copy of this license, visit http://creativecommons. org/licenses/by/4.0/. 


\section{References}

1. Bruix J, Gores GJ, Mazzaferro V. Hepatocellular carcinoma: clinical frontiers and perspectives. Gut. 2014;63:844-55.

2. Kreso A, Dick JE. Evolution of the cancer stem cell model. Cell Stem Cell. 2014;14:275-91.

3. Zhu PP, Fan ZS. Cancer stem cell niches and targeted Interventions. Prog Biochem Biophys. 2017;44:697-708.

4. Visvader JE, Lindeman GJ. Cancer stem cells: current status and evolving complexities. Cell Stem Cell. 2012;10:717-28.

5. Pastrana E, Silva-Vargas V, Doetsch F. Eyes wide open: a critical review of sphere-formation as an assay for stem cells. Cell Stem Cell. 2011;8:486-98.

6. Chiba T, Kita K, Zheng YW, Yokosuka O, Saisho H, Iwama A, et al. Side population purified from hepatocellular carcinoma cells harbors cancer stem cell-like properties. Hepatology. 2006;44:240-51.

7. Ho MM, Ng AV, Lam S, Hung JY. Side population in human lung cancer cell lines and tumors is enriched with stem-like cancer cells. Cancer Res. 2007;67:4827-33.

8. Haraguchi N, Ishii H, Mimori K, Tanaka F, Ohkuma M, Kim HM, et al. CD13 is a therapeutic target in human liver cancer stem cells. J Clin Invest. 2010;120:3326-39.

9. Ma S, Chan KW, Lee TKW, Tang KH, Wo JYH, Zheng BJ, et al. Aldehyde dehydrogenase discriminates the CD133 liver cancer stem cell populations. Mol Cancer Res. 2008;6:1146-53.

10. Yang ZF, Ho DW, Ng MN, Lau CK, Yu WC, Ngai P, et al. Significance of CD90(+) cancer stem cells in human liver cancer. Cancer Cell. 2008;13:153-66.

11. Zhu P, Wang Y, He L, Huang G, Du Y, Zhang G, et al. ZIC2dependent OCT4 activation drives self-renewal of human liver cancer stem cells. J Clin Invest. 2015;125:3795-808.

12. Chen ZZ, Huang L, Wu YH, Zhai WJ, Zhu PP, Gao YF. LncSox4 promotes the self-renewal of liver tumour-initiating cells through Stat3-mediated Sox4 expression. Nat Commun. 2016;7:12598.

13. Takebe N, Miele L, Harris PJ, Jeong W, Bando H, Kahn M, et al. Targeting Notch, Hedgehog, and Wnt pathways in cancer stem cells: clinical update. Nat Rev Clin Oncol. 2015;12:445-64.

14. Zhu P, Wang Y, Wu J, Huang G, Liu B, Ye B, et al. LncBRM initiates YAP1 signalling activation to drive self-renewal of liver cancer stem cells. Nat Commun. 2016;7:13608.

15. Zhu P, Wang Y, Du Y, He L, Huang G, Zhang G, et al. C8orf4 negatively regulates self-renewal of liver cancer stem cells via suppression of NOTCH2 signalling. Nat Commun. 2015;6:7122.

16. Yang W, Yan HX, Chen L, Liu Q, He YQ, Yu LX, et al. Wnt/ beta-catenin signaling contributes to activation of normal and tumorigenic liver progenitor cells. Cancer Res. 2008;68:4287-95.

17. Clevers H, Nusse R. Wnt/beta-catenin signaling and disease. Cell. 2012;149:1192-205.

18. MacDonald BT, Tamai K, He X. Wnt/beta-catenin signaling: components, mechanisms, and diseases. Dev Cell. 2009;17:9-26.

19. Hidaka S. Conflicting effects by antibodies against connexin 36 during the action of intracellular cyclic-AMP onto electrical synapses of retinal ganglion cells. $\mathrm{J}$ Integr Neurosci. 2016;15:571-91.

20. Li VSW, Ng SS, Boersema PJ, Low TY, Karthaus WR, Gerlach JP, et al. Wnt signaling through inhibition of beta-catenin degradation in an intact Axin1 complex. Cell. 2012;149:1245-56.

21. Lien WH, Fuchs E. Wnt some lose some: transcriptional governance of stem cells by Wnt/beta-catenin signaling. Gene Dev. 2014;28:1517-32.

22. Korinek V, Barker N, Willert K, Molenaar M, Roose J, Wagenaar $\mathrm{G}$, et al. Two members of the Tcf family implicated in Wnt/betacatenin signaling during embryogenesis in the mouse. Mol Cell Biol. 1998;18:1248-56.
23. MacDonald BT, He X. Frizzled and LRP5/6 receptors for Wnt/ beta-catenin signaling. Cold Spring Harb Perspect Biol. 2012;4: a007880..

24. De Marco P, Merello E, Rossi A, Piatelli G, Cama A, Kibar Z, et al. FZD6 is a novel gene for human neural tube defects. Hum Mutat. 2012;33:384-90.

25. Naz G, Pasternack SM, Perrin C, Mattheisen M, Refke M, Khan $\mathrm{S}$, et al. FZD6 encoding the Wnt receptor frizzled 6 is mutated in autosomal-recessive nail dysplasia. Brit $\mathrm{J}$ Dermatol. 2012;166:1088-94.

26. Golan T, Yaniv A, Bafico A, Liu GZ, Gazit A. The human frizzled 6 (HFz6) acts as a negative regulator of the canonical Wnt center dot beta-catenin signaling cascade. J Biol Chem. 2004;279:14879-88.

27. Lyons JP, Mueller UW, Ji H, Everett C, Fang X, Hsieh JC, et al. Wnt- 4 activates the canonical beta-catenin-mediated Wnt pathway and binds frizzled-6 CRD: functional implications of Wnt/betacatenin activity in kidney epithelial cells. Exp Cell Res. 2004;298:369-87.

28. Batista PJ, Chang HY. Long noncoding RNAs: cellular address codes in development and disease. Cell. 2013;152:1298-307.

29. Wang $P$, Xue YQ, Han YM, Lin L, Wu C, Xu S, et al. The STAT3-binding long noncoding RNA lnc-DC controls human dendritic cell differentiation. Science. 2014;344:310-3.

30. Yuan JH, Yang F, Wang F, Ma JZ, Guo YJ, Tao QF, et al. A long noncoding RNA activated by TGF-beta promotes the invasionmetastasis cascade in hepatocellular carcinoma. Cancer Cell. 2014;25:666-81.

31. Tseng YY, Moriarity BS, Gong W, Akiyama R, Tiwari A, Kawakami H, et al. PVT1 dependence in cancer with MYC copynumber increase. Nature. 2014;512:82-86.

32. Leucci E, Vendramin R, Spinazzi M, Laurette P, Fiers M, Wouters $\mathrm{J}$, et al. Melanoma addiction to the long non-coding RNA SAMMSON. Nature. 2016;531:518-22.

33. Yang F, Zhang H, Mei Y, Wu M. Reciprocal regulation of HIF1alpha and lincRNA-p21 modulates the Warburg effect. Mol Cell. 2014;53:88-100.

34. Wang KC, Chang HY. Molecular mechanisms of long noncoding RNAs. Mol Cell. 2011;43:904-14.

35. Roessler S, Jia HL, Budhu A, Forgues M, Ye QH, Lee JS, et al. A unique metastasis gene signature enables prediction of tumor relapse in early-stage hepatocellular carcinoma patients. Cancer Res. 2010;70:10202-12.

36. Roessler S, Long EL, Budhu A, Chen Y, Zhao X, Ji J, et al. Integrative genomic identification of genes on $8 p$ associated with hepatocellular carcinoma progression and patient survival. Gastroenterology. 2012;142:957-66. e912

37. Henderson BR. Nuclear-cytoplasmic shuttling of APC regulates beta-catenin subcellular localization and turnover. Nat Cell Biol. 2000;2:653-60.

38. Ponting CP, Oliver PL, Reik W. Evolution and functions of long noncoding RNAs. Cell. 2009;136:629-41.

39. Luo S, Lu JY, Liu L, Yin Y, Chen C, Han X, et al. Divergent lncRNAs regulate gene expression and lineage differentiation in pluripotent cells. Cell Stem Cell. 2016;18:637-52.

40. Zhu P, Wang Y, Huang G, Ye B, Liu B, Wu J, et al. Inc-betaCatm elicits EZH2-dependent beta-catenin stabilization and sustains liver CSC self-renewal. Nat Struct Mol Biol. 2016;23:631-9.

41. Helming KC, Wang XF, Roberts CWM. Vulnerabilities of mutant SWI/SNF complexes in cancer. Cancer Cell. 2014;26:309-17.

42. Phelan ML, Sif S, Narlikar GJ, Kingston RE. Reconstitution of a core chromatin remodeling complex from SWI/SNF subunits. Mol Cell. 1999;3:247-53. 